OAK RIDGE NATIONAL LABORATORY

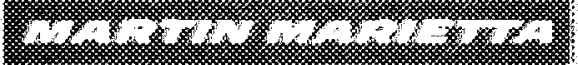

\section{Characterization Plan for the Old Hydrofracture Facility}

\author{
C. W. Francis \\ R. G. Stansfiod
}

ENVMOMMEWTAL SCHENCES DNGOM PUBLICATION MO 2701

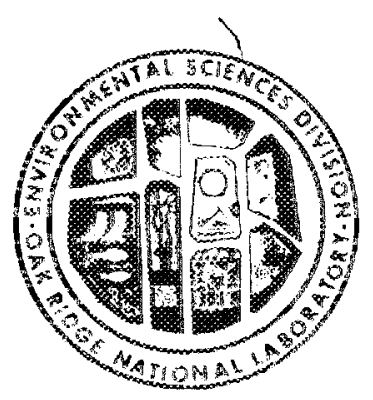

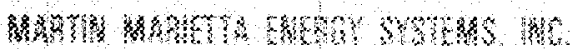

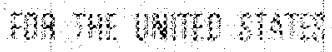

nemmbmen who 
Frinted in the United States of America Available from National Technical Information Service U.S. Depariment of Commerco 5285 Port Royal Road, Springfield, Virginia 22161 NTIS price codes - Printed Copy: A05 Microfiche A0?

This report was prepared as an account of work sponsored by an agency of the United States Government. Neither the United States Government nor any agency thereof, noi any of their employees, makes any warranty, express or implied, or assuines any legal liability or responsibility for the accuracy. compleieness, or usefulness of any information, apparatus, product, or process disclosed, or represents that its use would not infringe privately owned rights. Reference herein to any specific commercial product, process, or service by tradename, iradenark, manufaciurer, or otherwise, does not necessarly constitute or imply its endorsement, recommendation, or favoring by the United States Government or any agency thereof. The views and op inions of authors expressed herein do not necessarily state or reflect those of the United States Government or any agency thereot. 
NUCLEAR AND CHEMICAL WASTE PROGRAM

(Activity No. AR 0510100 ; ONL-WD20)

Date Published: September 1986

Prepared for the

office of Defense Waste and Transportation Management

Prepared by the OAK RIOGE NATIONAL LABORATORY Oak Ridge, Tennessee 37831 operated by

MARTIN MARIETTA ENERGY SYSTEMS, INC. for the

U.S. DEPARTMENT OF ENERGY

under Contract No. DE-ACO5-840R21400 



\section{CONTENTS}

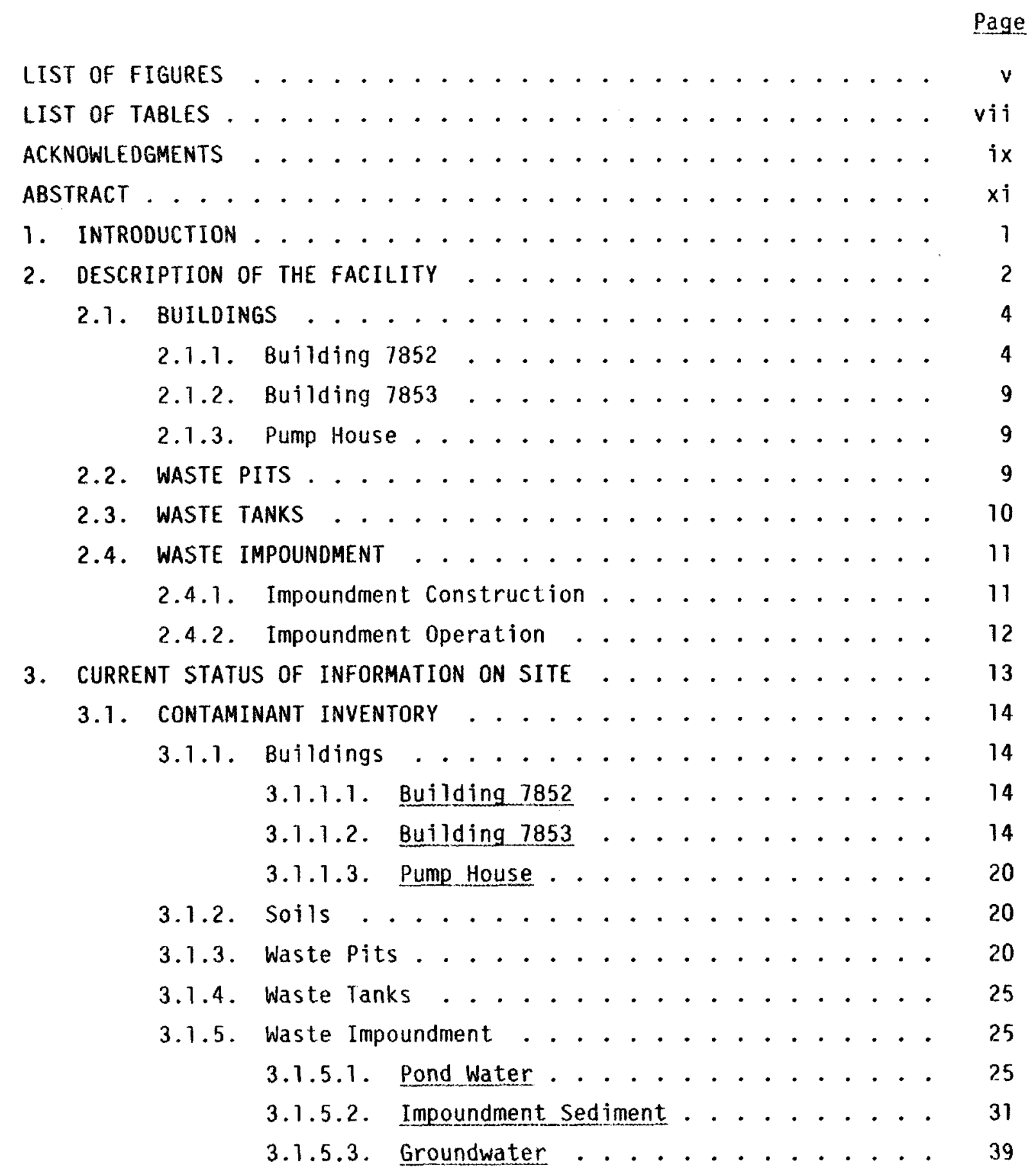




\section{Page}

3.2. GEOLOGY AND SOILS . . . . . . . . . . . . . . 45

3.2.1. Regional Geology............... . 45

3.2.2. Site Geology................ . 45

3.2.2.1. Bedrock ............. . 45

3.2.2.2. Soils . . . . . . . . . 48 48

3.3. HYDROLOGY . . . . . . . . . . . . . . . 48

3.3.1. Groundwater Movement . . . . . . . . . 50

3.3.2. Uppermost Aquifer. . . . . . . . . . . 53

3.4. ECOLOGY .................... . . 54

4. ADOITIONAL INFORMATION NEEDEO . . . . . . . . . . . . . . 55

4.1. CONTAMINANT INVENTORY . . . . . . . . . . . . . . 55

4.1.1. Buildings. . . . . . . . . . . . . . 55

4.1.1.1. Building 7852. . . . . . . . . 55

4.1.1.2. Building 7853........... . 57

4.1.1.3. Pump House . . . . . . . . . . 57

4.1.2. Waste Pits................. 58

4.1.3. Waste Tanks ................. 58

4.1.4. Waste Impoundment .............. 59

4.2. GEOLOGY AND SOILS ................. . . 59

4.2.1. Site Geology.............. . . 59

4.2.2. Soils ................. . . 60

4.3. HYDROLOGY ..................... . . 61

4.3.1. Surface Water. . . . . . . . . . . . . . . 61

4.3.2. Groundwater ............... 62

4.4. ECOLOGY . . . . . . . . . . . . . . . . . . 63

5. MONITORING ANO TeSting PLAN . . . . . . . . . . . . . . . . 65

5.1. RECOMMENDED ACTIONS TASKS. . . . . . . . . . . . 65

6. REFERENCES .......................... . . . . 69 


\section{LIST OF FIGURES}

Figure

Page

1 Location of old hydrofracture facility at the Oak Ridge National Laboratory. . . . . . . . 3

2 Site plan of the old hydrofracture facility ...... 5

3 Photograph of the old hydrofracture facility ...... 6

4 Radiological survey results of the control room .... 15

5 Radiological survey results of the mixing cell ..... 16

6 Radiological survey results of the pump cell . . . . . 17

7 Radiological survey results of the well cell ...... 18

8 Radiological survey results of the engine pad . . . . 19

9 Radiological survey results of the pump room . . . . . 21

10 Beta-gamna direct readings exceeding

$0.3 \mathrm{mrad} / \mathrm{h}$ at 1 to $3 \mathrm{~cm}$ above the surface ....... 22

11 Location of sediment sampling sites and groundwater monitoring wells.......... 33

12 Geologic map of the ORNL area including the old hydrofracture facility............ . 46

13 Geologic section through the old hydrofracture facility impoundment............... 49

14 General water-table map of the area upgradient from the old hydrofracture facility. . . . . 51

15 Water-table map of the old hydrofracture facility impoundment................ 



\section{LIST OF TABLES}

Tables

Page

1 Dimensions for rooms in building 7852 . . . . . . . 8

2 Gamma screening of deep soil cores........... 23

3 Waste pit inventories of radionuclides . . . . . . . 24

4 Estimate of residual radioactivity in waste tanks at the old hydrofracture facility . . . . . 26

5 Concentration and inventory of nonradioactive contaminants in OHF pond water........... 27

6 Concentration and inventory of organic chemicals in $\mathrm{OHF}$ pond water . . . . . . . 28

7 Concentration and inventory of radionuclides in $0 H F$ pond water . . . . . . . . . . 29

8 Concentration of RCRA regulated constituents in EP extracts of sediment from the OHF impoundment . . . . 34

9 Concentration and inventory of nonradioactive contaminants in sediment from the OHF impoundment . . . 36

10 Concentration and inventory of radionuclides in sediment from the OHF impoundment......... 38

11 Summary of monitoring well location, construction data, and water levels at the OHF impoundment ......

12 Groundwater concentrations in wells downgradient from the OHF impoundment . . . . . . . . 



\section{ACKNOWLEDGMENTS}

We wish to express our thanks to W. J. Boegly, Jr., who coordinated this work and gave us some useful guidance in the planning and reviewing stages. We greatly appreciated the help provided to us by D. R. Simpson for the Environmental and Occupational Safety Division with respect to identifying the potential radiological hazards and needed tasks required at the control buildings and pump house. We appreciated the suggestions made in the review comments by B. P. Spalding and D. D. Huff. In the same tone, we wish to thank B. W. Burwinkle and T. E. Myrick for their interest and comments on the early drafts of this document. 



\section{ABSTRACT}

FRANCIS, C. W., and R. G. STANSFIELD. 1986. Characterization Plan for the 01d Hydrofracture Facility. ORNL/TM-9991. Oak Ridge National Laboratory, Oak Ridge, Tennessee. $91 \mathrm{pp}$.

U. S. Department of Energy (DOE) facilities are required to comply fully with all federal and state regulations. In response to this requirement, the Oak Ridge National Laboratory (ORNL) has established the remedial action program, to provide comprehensive management of areas where past research, development, and waste management activities have been conducted and have resulted in residual contamination of facilities or the environment. One of the objectives of this program is to define the extent of contamination at these sites. The intent is to document the known environmental characteristics of the sites and identify the additional actions, such as sampling, analytical measurements, and modeling, necessary to confirm contamination and the possible migration of contaminants from the sites. One of these sites is the 01d Hydrofracture Facility (OHF).

The OHF was used for the permanent disposal of liquid radioactive waste in impermeable shale formations at depths ranging from about 230 to $300 \mathrm{~m}$ ( 750 to $1000 \mathrm{ft}$ ), from 1964 to 1979 . The liquid waste was blended into a pumpable grout by mixing it with cement and special clays used to immobilize radionuclides against groundwater transport. This report summarizes the results of several studies at ORNL that have measured the concentration of radionuclides and, to some extent, concentrations of hazardous chemicals in the sediment of the impoundment, as well as the concentrations in soils and groundwater 
near the facility. The report addresses only the contamination of and the potential releases to the environment that might result from the facility per se and makes no attempt to address potential releases that might result from permanent disposal of wastes (i.e., the grout sheets) during its operation. Outlined in the report are the additional actions needed to obtain the information required to confirm the extent of contamination within the facility. The major efforts include the measurement of radionuclides and potentially hazardous chemicals contained in the five underground waste storage tanks at the facility and determining of the lateral and vertical contamination due to seepage of waste from the impoundment. 


\section{INTRODUCTION}

U. S. Department of Energy (DOE) facilities are required to comply fully with all federal and state regulations. In response to an application to the U. S. Environmental Protection Agency (USEPA) by the Oak Ridge National Laboratory (ORNL) for a permit to operate a hazardous waste storage facility, the USEPA has required ORNL to comply with the $3004(u)$ provision of the 1984 Hazardous and Solid waste Amendment of the reauthorization of the Resource Conservation and Recovery Act (RCRA). Under these regulations the Permittee is required to identify and characterize all solid waste managenent units currently or previously located within ORNL's boundary. The intent of this regulation is to determine whether a prior or continuing release of hazardous waste or hazardous constituents have occurred and/or to characterize the nature and extent of the releases.

One of the facilities that may pose an undue risk to health, safety, and environment as a result of the migration of hazardous substances to groundwater is the old Hydrofacture Facility (OHF).

This report documents the existing environmental information on the OHF and defines additional actions, such as the installation of monitoring wells, collection of samples, and analytical measurements, required to confirm contamination and possible migration of contaminants from the site. Also included are descriptions of activities required to collect additional geologic and hydrologic information necessary to model the site performance. 
ORNL/TM-9991

\section{DESCRIPTION OF THE FACILITY}

The old hydrofracture facility (OHF) was used for the permanent disposal of liquid radioactive waste in impermeable shale formations at depths ranging from approximately 229 to $300 \mathrm{~m}(750$ to $1000 \mathrm{ft}$ ) from 1964 to 1979 (weeren 1976 and weeren 1980). The liquid waste was formed into a pumpable grout by blending with cement and special clays used to immobilize radionuclides against groundwater transport. The grout was injected through a slotled well casing into the shale where it solidified into nearly horizontally thin sheets, depending on the orientation of the shale forination, around the injection well in a generally elliptical pattern. The average concentration of radionuclides in the grout mixture prior to injection was approximately $10 \mathrm{MBq} / \mathrm{mL}(0.26 \mathrm{mCi} / \mathrm{mL})$ or less for beta-gamma-emitting radionuclides and $370 \mathrm{~Bq} / \mathrm{g}(10 \mathrm{nCi} / \mathrm{g})$ or less for transuranic alpha emitting radionuclides. For a single injection, as much as $7.6 \times 10^{6} \mathrm{~L}$ $\left(2 \times 10^{6}\right.$ gal $)$ of waste grout containing as much as $2.2 \times 10^{4} \mathrm{GBq}$ $\left(6.0 \times 10^{5} \mathrm{ci}\right)$ were disposed of.

The OHF is located approximately 1.1 miles southwest of the main complex of the Oak Ridge National Laboratory at the confluence of Melton Branch and White oak creeks (see Fig. 1). Immediately to the east of the OHF is the western fenced boundary of the solid waste Storage Area 5 (SWSA 5), where low-level radioactive solid wastes were 
ORNL-DWG 85-13767R

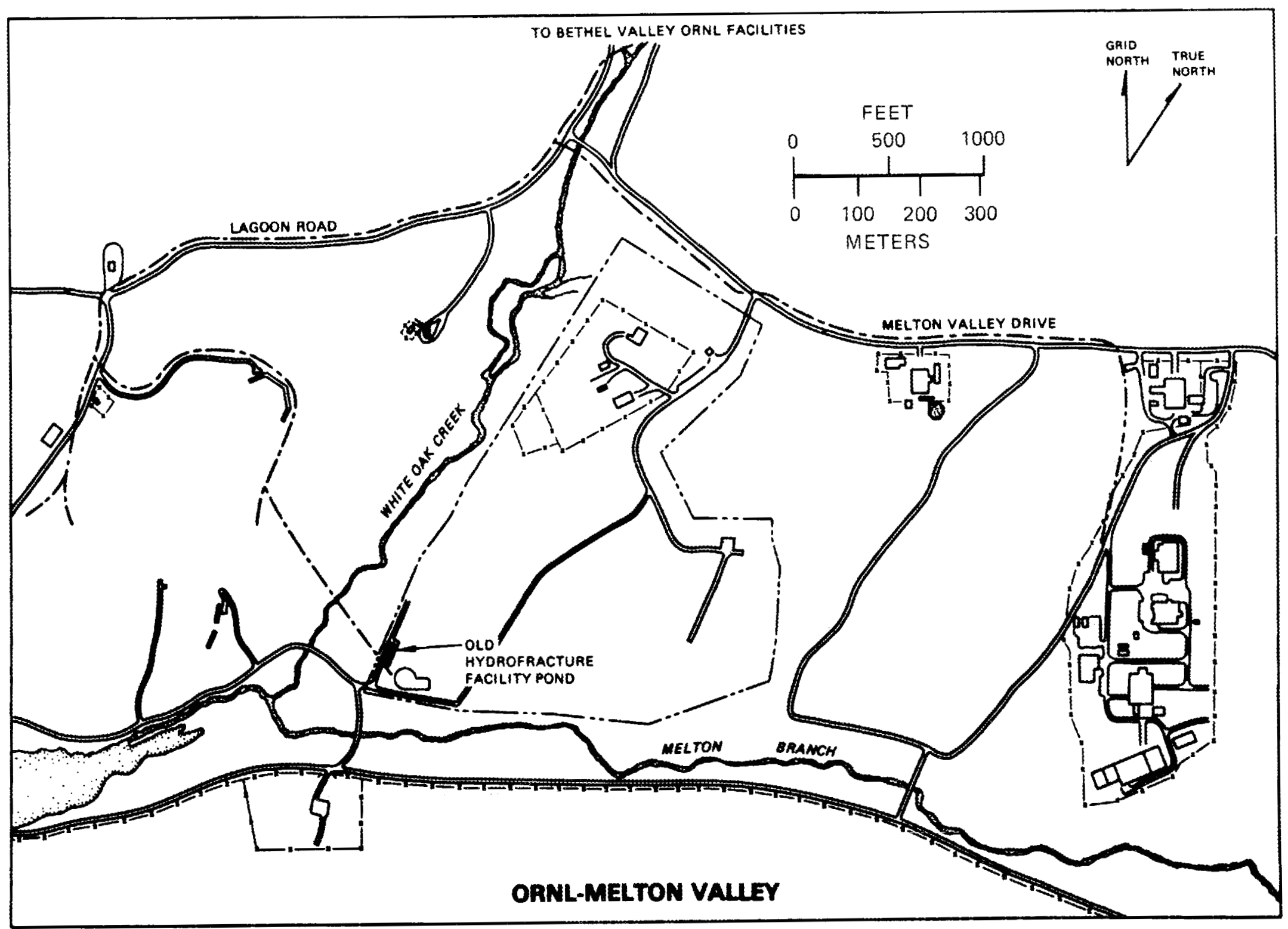

$\omega$

Fig. 1. Location of old hydrofracture facility at the Oak Ridge National Laboratory. 
buried between 1958 and 1973. The facility is approximately $120 \mathrm{~m}$ $(400 \mathrm{ft}$ ) east of White 0ak Creek and approximately the same distance north of the Melton Branch. White Oak Creek flows into white Oak Lake and hence into the clinch River.

The OHF site consists of three buildings (Buildings 7852, 7853, and a pump house) and some waste related containment facilities (waste pits, tanks, and an impoundment that was constructed to serve as an emergency containment basin in the event of a spill from the radioactive grout injections. The site plan and a photograph of the OHF are illustrated in Figs. 2 and 3 , respertively.

The focus of this plan is limited to the buildings, equipment, and waste related facilities near surface, such as the waste pits, waste tanks and the waste impoundment. It does not address the characterization of disposed waste injected during the facility's lifetine.

\subsection{BUILOINGS}

\subsubsection{Building 7852}

Building 7852 contains a mixing cell, a pump cell for the head end of the injection pump, a well cell, a transit-roof-covered engine pad (at the south end), and a control room (at the north end). The three cells, which were used for the mixing, pumping, and injection of the grout, have a $30-\mathrm{cm}(12-$ in) thick concrete walls and are covered with a metal roof (triple-layered steel, two $1 / 4-i n$. plates on either side of a steel grating). The walls, however, are only painted and are not lined with metal as is the ceiling. The hot cells have windows 


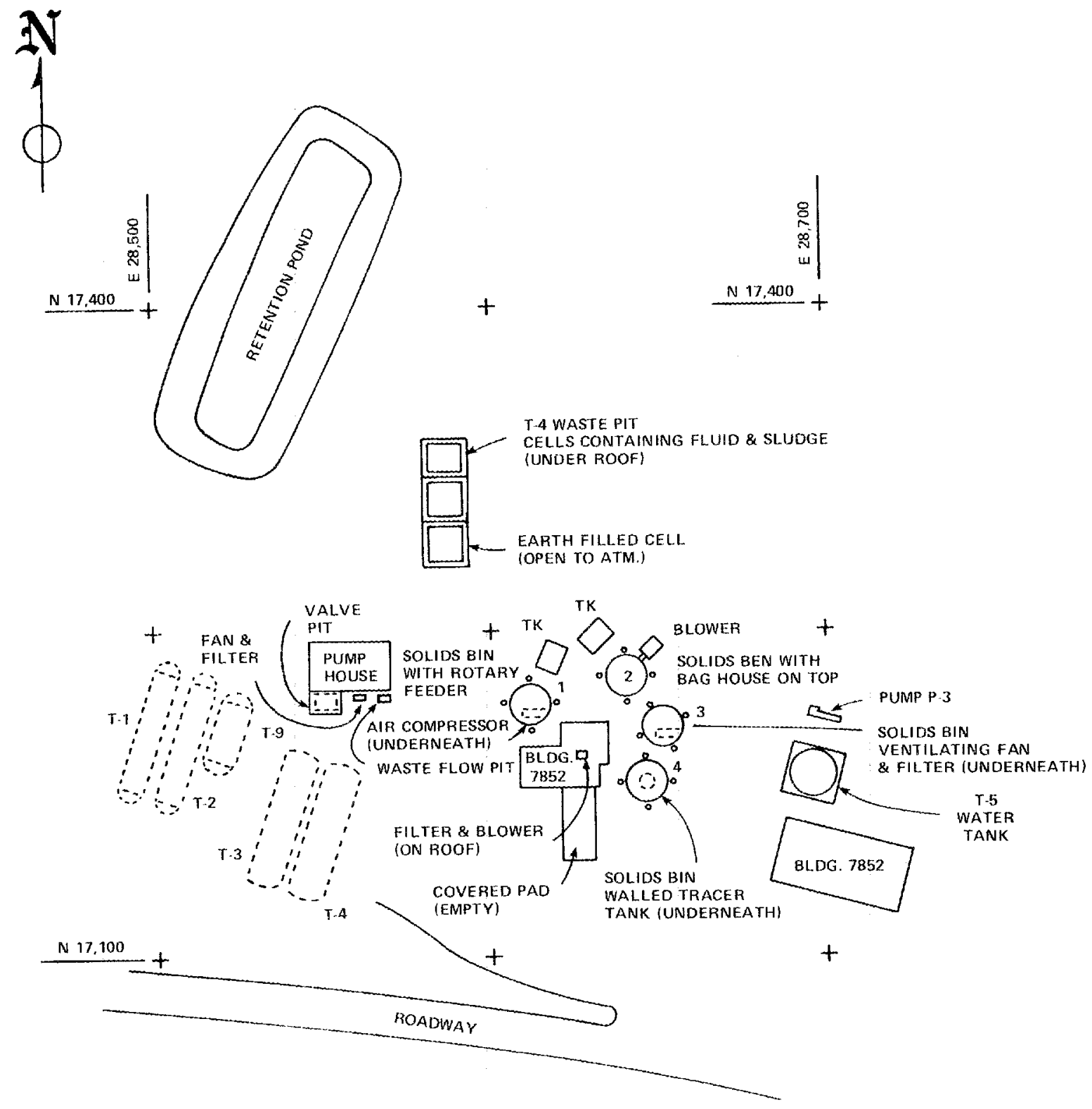

Fig. 2. Site plan of the old hydrofracture facility. 
ORNL-PHOTO 0033-78

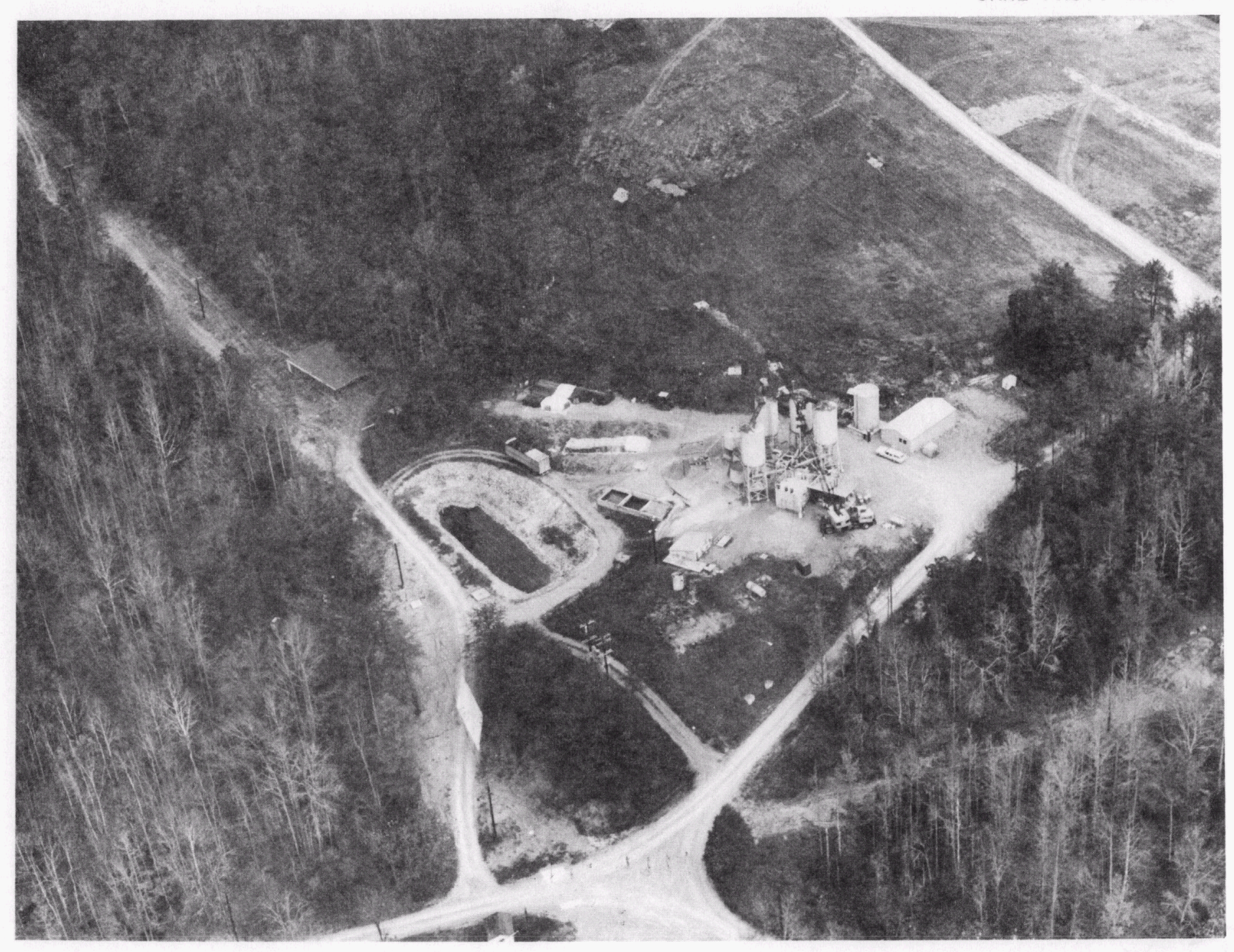

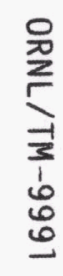

o

Fig. 3. Photograph of the old hydrofracture facility. 
constructed of bulletproof glass. The roof of the mixing cell is fixed in place, but the roofs of the pump cell and the well cell can be removed. The control room roof is metal decking. on the roof of building 7852 are a hoist, an air filter and blower, and three disconnected solids conveyers used to transport solids from the adjacent bulk storage bins. Listed in Table 1 are the dimensions for building 7852 .

Within the mixing cell are located a mixer assembly and a grout mix "tub." The mixer assembiy is approximately $2.7 \mathrm{~m}(9 \mathrm{ft}) \mathrm{high}$ and has the shape of a cone that is $1.5 \mathrm{~m}(5 \mathrm{ft})$ in diameter at the top. The grout "tub" is approximately $1 \mathrm{~m}(3 \mathrm{ft})$ in diameter and $2 \mathrm{~m}$ $(6.5 \mathrm{ft})$ high. The tops of both of these vessels and the motor for the agitation of the grout mix extend through the roof of the mixing cell. The cell also contains valves and piping.

The well cell contains a small drum-sized tank, piping, valves, and the top of the injection we 11. The pump cell also contains some equipment, namely, hoses, piping, and valves.

Around building 7852 are located four cylindrical bulk storage bins with conical bottoms (see Fig. 2). These are elevated to permit gravity flow of the solids (cement, fly ash, clay, and other additives used during the process) through air slides to building 7852 . Only one air slide is now connected to the mix hopper in building 7852 . Each bin is $3.6 \mathrm{~m}(12 \mathrm{ft})$ in diameter by $6 \mathrm{~m}(20 \mathrm{ft})$ high. The bins are interconnected by piping and by a catwalk. Under bin 4 is a small vessel once used to contain a short-lived radioactive tracer fluid; concrete shielding blocks surround this vessel. Directly to the east 
Table 1. Dimensions for rooms in building 7852

$\begin{array}{lccr}\text { Room } & \begin{array}{c}\text { Length } \\ (\mathrm{m})\end{array} & \begin{array}{c}\text { Width } \\ (\mathrm{m})\end{array} & \begin{array}{c}\text { Height } \\ (\mathrm{m})\end{array} \\ \text { Mixing cell } & 3.8 & 3.5 & 2.4 \\ \text { Pump cell } & 3.0 & 2.3 & 2.4 \\ \text { Well cell } & 3.3 & 3.4 & 3.0 \\ \text { Control room } & 4.4 & 3.3 & 3.1 \\ \text { Engine pad } & 9.1 & 3.0 & 2.7-3.0\end{array}$


of building 7852 is a 97,000-L (25,000-gal) water tank and pump for delivering water to the facility during mixing and injection.

\subsubsection{Building 7853}

This building was used as a change room for operators of the OHF during grout injections. It is now used for storage.

\subsubsection{Pump House}

The pump house, located to the northwest of building 7852 , contains two 30-hp progressive-cavity-type pumps that were used to feed waste from the nearby buried storage tanks, via underground piping, to building 7852 . This $33-\mathrm{m}^{2}\left(360-\mathrm{ft}^{2}\right)$ concrete block house was built in an excavation with only the roof and southeast corner (at the door) being fully exposed. A valve pit [6 ft $4 \mathrm{in}$. by $21 \mathrm{ft}(1.9$ by $6.4 \mathrm{~m})]$ is located at the southwest corner of the pump house. This pit is covered with metal plates through which valve handles extend for opening and closing valves.

\subsection{WASTE PITS}

Approximately $6.2 \mathrm{~m}(20 \mathrm{ft})$ to the northeast of the pump house are the waste pits that are composed of three separate concrete-walled cells (12 by $12 \mathrm{ft}$ wide and approximately $9 \mathrm{ft}$ deep, $3.7 \times 3.7 \times 2.7 \mathrm{~m}$ ). These pits were used to allow maxinum recycle of contaminated water during slotting and wash-up, minimizing the need to inject waste water. The original pit, the southern cell, was filled with grout during an experimental injection, and two additional cells were built to the north. These two pits now contain water-covered sludge. The 
two northern cells are covered with a corrugated plastic roof, while the south cell remains uncovered.

\subsection{WASTE TANKS}

Five buried carbon steel tanks were used for storing liquid radioactive waste prior to injection by the OHF. These tanks are located about $18 \mathrm{~m}(60 \mathrm{ft})$ directly west of building 7852 . These buried storage tanks, which were placed horizontally, are still connected to the Intermediate-Level Waste (ILW) transfer line, allowing the tanks to serve as a possible emergency storage site. There remains in each of the tanks approximately a foot $(30 \mathrm{~cm})$ of residual ILW, amounting to less than $10 \%$ of the total volume in the tanks. The tanks were installed on concrete pads in open pits that were equipped with concrete dividing walls to separate the tanks and allow for monitoring of possible leakage. The tanks, which are covered with approximately $1.2 \mathrm{~m}(4 \mathrm{ft})$ of soil, are under cathodic protection and vented through a HEPA filter to the atinosphere. The voltage/amperage applied to the cathodic protection system and the general radiation background of the storage tank dry well systems (monitoring wells located within the concrete enclosure built to contain each of the tanks) are monitored daily. The ventilation system is also checked daily, and any accumulated liquids in the storage tank dry well systems are sampled and analyzed for radioactivity. 


\subsection{WASTE IMPOUNDMENT}

\subsubsection{Impoundment Construction}

The impoundment was constructed as part of the hydrofracture operation in 1963, by excavating a rectangular basin into the base of the valley wall. Construction dimensions of the bottom of the basin are $6 \mathrm{~m}(20 \mathrm{ft})$ in width by $30 \mathrm{~m}(100 \mathrm{ft})$ in length, with sides sloping at 1 vertical on 1.5 horizontal. The depth of the impoundment is slightly greater than $1.5 \mathrm{~m}(5 \mathrm{ft}$ ) at the low (west) side. The sides are lined with limestone rip-rap. Design capacity was $379,000 \mathrm{~L}$ $(100,000 \mathrm{gal})$. Inflow was to the south end of the impoundment via a buried $46-c m(18-i n$.$) diam line from the injection well cell. A 20-cm$ (8-in.) diam line from the waste pits upslope from the impoundment is also shown on drawings as entering the impoundment at the same location. Construction drawings specified that the impoundment bottom be sprayed with liquid asphalt to control erosion, and a plastic liner was placed in the impoundment prior to experimental injections. However, no evidence of either of these treatments was observed by Stansfield and Francis (1986) while sampling the sediment. A 1.5-m $(5-f t)$ high, concrete standpipe was provided as an emergency outflow at the north end of the impoundment. ORNL drawing S-10,916 EA 001 D shows this vertical standpipe connected to an 8 -in $(20-\mathrm{cm})$ vitrified clay, pipeline. The drawing also shows this line extending to the west for a distance of approximately $15 \mathrm{~m}(50 \mathrm{ft})$ where it empties into a shallow, natural swale at an approximate elevation of $233 \mathrm{~m}(763 \mathrm{ft})$. Probings made by Stansfield and Francis (1986) indicate that the bottom of the impoundment is at an approximate elevation of $231.1 \mathrm{~m}(764.6 \mathrm{ft})$. 


\subsubsection{Impoundment Operation}

The impoundinent was constructed to serve as an emergency contaimment vasin in the event of a spill from the radioactive grout injections, for example, one caused by back-flow of grout. Due to malfunction of pumping equipment or piping, the impoundment did receive radioactive grout from injections made in 1965 (de Laguna et al. 1971) and 1971 (L. C Lasher, Operations Division, personal communication). Prior to a grout injection at the facility, the water level in the impoundinent was required to be low enough that there would be sufficient capacity in the impoundment to hold the radioactive grout should an emergency arise that required such action during the operation. Prior to some injections, depending on the water level, this necessitated decanting the water from the impoundment. Before contanination of the impoundment by radioactive waste, the pond water was siphoned to the white Oak Creek flood plain. Subsequent to contamination of the impoundment, the water was pumped to the low-level waste system for processing (L. C Lasher, oral communication, 1985). Operation of the OHF facility ceased by 1980 (Myrick 1984). In the winter of 1984-85, the impoundment received drilling fluid and drill cuttings from an exploratory core boring $(5.7-\mathrm{cm}-\mathrm{diam}$ core and 8.6-cin-dian hole) through the radioactive grout sheets underlying the OHF site. Probings made by Stansfield and Francis (1986) indicate that the thickness of the sediment in the impoundment averages $27 \mathrm{~cm}(0.9 \mathrm{ft})$. This amounts to approximately $55,000 \mathrm{~L}(14,500$ gal) of sediment. 


\section{CURRENT STATUS OF INFORMATION ON SITE}

Considerable site specific, environmental information on the facility exists in published reports (McMaster and Waller 1965; Stansfield and Francis 1986), In addition, S. F. Huang [Environmental and Occupational Safety Division (EOSD), personal communication, September 1984] has conducted considerable radiological and chemical andys is at the facility.

\subsection{CONTAMINANT INVENTORY}

\subsubsection{Buildings}

A preliminary radiological survey was completed on the buildings by S. F. Huang (EOSU, personal communication, September 1984). Standard ORNL radiation survey instruments were used for all surveys. Beta-ganma readings were made with a GM neter, a victoreen 440 (a low-range air ionization chamber), or a cutie Pie (Gupton 1961). Smear

samples were taken over areas of approximately $100 \mathrm{~cm}^{2}$ and counted in alpha and beta-gamma sample counters or with a portable survey instrument for the samples with high levels of contamination.

\subsubsection{Building 7852}

Radiation and contamination levels in the interiors of the control room, mixing ce11, pump ce11, well cel1, and pump room were measured. To measure the high levels of transferable contamination on the rough surfaces of the interior walls, wet paper towel smears were used. These smears were surveyed with a portable instrument rather than with smear counters to prevent contanination of the smear counters. 
In the control room, the absorbed dose rates ranged from 0.75 to $6 \mathrm{mgy} / \mathrm{h}(75$ to $600 \mathrm{mrad} / \mathrm{h})$. Smearable activity per $100 \mathrm{~cm}^{2}$ varied from 330 to $820 \mathrm{~Bq}(8.9$ to $22.3 \mathrm{nC} i)$ of beta-gamma and from 0.3 to $0.7 \mathrm{~Bq}(8$ to $22 \mathrm{pCi}$ ) of alpha (see Fig. 4). High levels of direct beta gamma readings ranging from 1.5 to $40 \mathrm{mGy} / \mathrm{h}(150$ to $4000 \mathrm{mrad} / \mathrm{h}$ ) were observed in the mixing, pump, and well cells. Removable beta-gamma activity measured from 0.05 to $0.35 \mathrm{mgy}$ ( 5 to $35 \mathrm{mrad}$ ). For the most part, removable alpha activity was less than $1.7 \mathrm{~Bq} / 100 \mathrm{~cm}^{2}$ $\left(<50 \mathrm{pci} / 100 \mathrm{~cm}^{2}\right)$ in these rooms (see Figs. 5,6 and 7$)$. At $10 \mathrm{~cm}$ above the engine pad, dose rates ranged from 0.2 to $3 \mathrm{mGy} / \mathrm{h}$ (20 to $300 \mathrm{mrad} / \mathrm{h}$ ). Reinovable beta-gama activity on the engine pad, using the wet towel smear technique previously described, varied from 5 to $10 \mu \mathrm{Gy} / \mathrm{h}(0.5$ to $1 \mathrm{mrad} / \mathrm{h})$ per $100 \mathrm{~cm}^{2}$. Alpha activity, using the same smear technique, was usually less than $0.5 \mathrm{~Bq}(14 \mathrm{pC} i)$ per $100 \mathrm{~cm}^{2}$ (see Fig. 8).

It is not clear how thoroughly S. F. Huang (EOSD, personal conmunication, September 1984) surveyed the four bulk storage bins or their connecting air slides, catwalk, etc. For example, it was stated in the S. F. Huang (EOSD, personal communication, September 1984) that these bins and the water tank were "considered to be relatively uncontaminaled, with no serious radiological impacts expected."

\subsubsection{Building 7853}

This building, which was used as a change room during OHF operations, was also "considered to be relatively uncontaminated, with no serious radiological impacts expected." 
ORNL-DWG 84-12881

CONTROL ROOM

(4)

(1)

$600 \mathrm{mrad} / \mathrm{h} * * *$

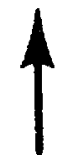

N

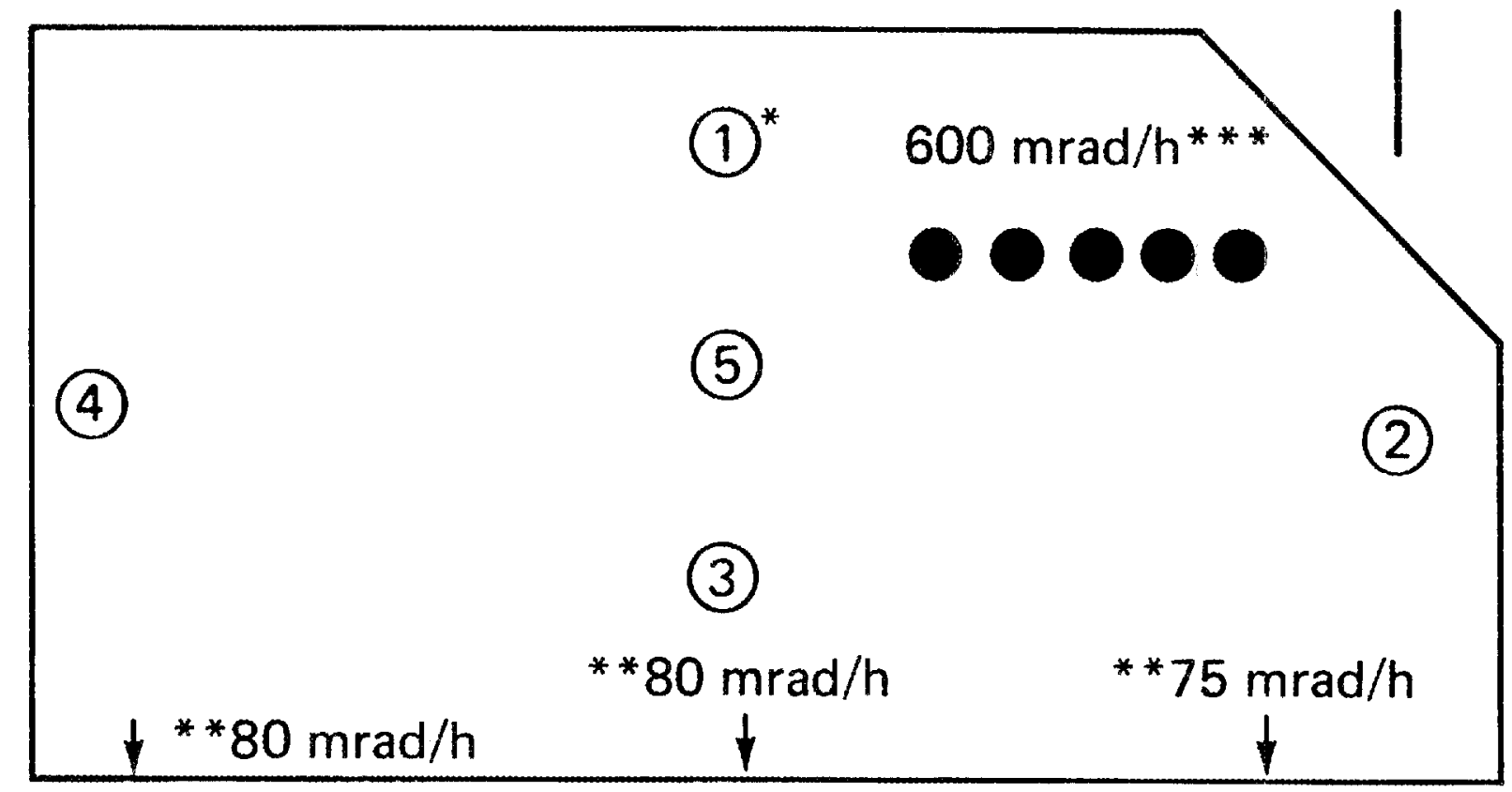

* SMEAR SAMPLE LOCATION

**READING AGAINST THE WALL

***READING FROM FIVE STORED ITEMS

SMEARABLE ACTIVITY

PER $100 \mathrm{~cm}^{2}$

\begin{tabular}{ccr}
\hline LOCATION & BETA-GAMMA & ALPHA \\
\hline 1 & $49,200 \mathrm{dpm}$ & $<20 \mathrm{dpm}$ \\
2 & $32,200 \mathrm{dpm}$ & $<20 \mathrm{dpm}$ \\
3 & $63,320 \mathrm{dpm}$ & $30 \mathrm{dpm}$ \\
4 & $27,600 \mathrm{dpm}$ & $21 \mathrm{dpm}$ \\
5 & $20,650 \mathrm{dpm}$ & $36 \mathrm{dpm}$
\end{tabular}

Fig. 4. Radiological survey results of the control room (1 mrad $=10 \mu G y)$. Direct beta-garnila readings are marked. 


\section{MIXING CELL}

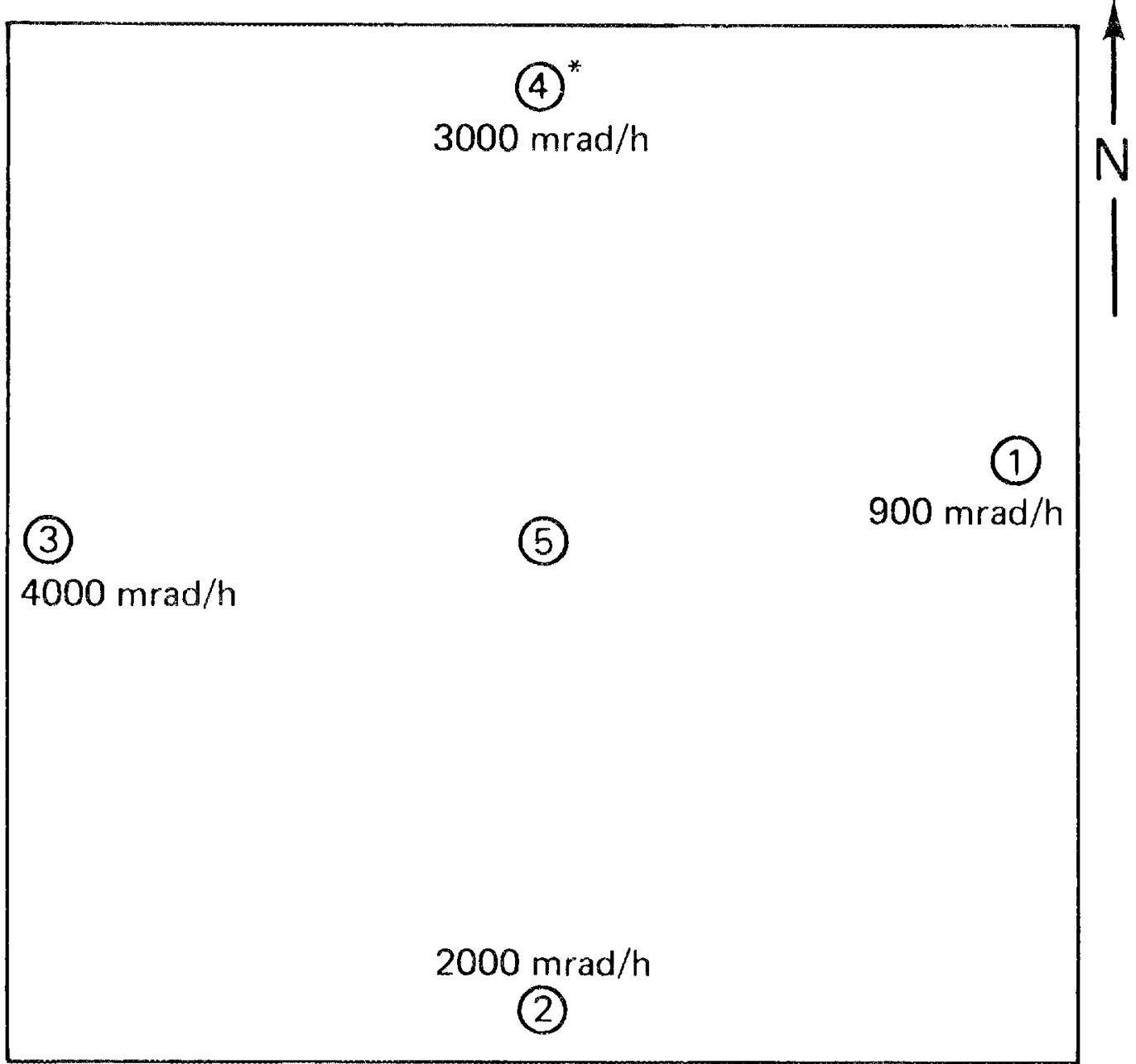

*SMEAR SAMPLE LOCATION

\begin{tabular}{crr}
\hline & \multicolumn{2}{c}{$\begin{array}{c}\text { SMEARABLE ACTIVITY } \\
\text { PER } 100 \mathrm{~cm}^{2}\end{array}$} \\
\hline LOCATION & BETA-GAMMA & ALPHA \\
\hline & $15 \mathrm{mrad} / \mathrm{h}$ & $102 \mathrm{dpm}$ \\
1 & $15 \mathrm{mrad} / \mathrm{h}$ & $<20 \mathrm{dpm}$ \\
3 & $15 \mathrm{mrad} / \mathrm{h}$ & $<20 \mathrm{dpm}$ \\
4 & $5 \mathrm{mrad} / \mathrm{h}$ & $<20 \mathrm{dpm}$ \\
4 & $30 \mathrm{mrad} / \mathrm{h}$ & $<20 \mathrm{dpm}$
\end{tabular}

Fig. 5. Radiological survey results of the mixing cell (1 mrad $=10 \mu \mathrm{Gy})$. birect beta-gamma readings are marked. 
ORNL_DWG 84-12879

\section{PUMP CELL}

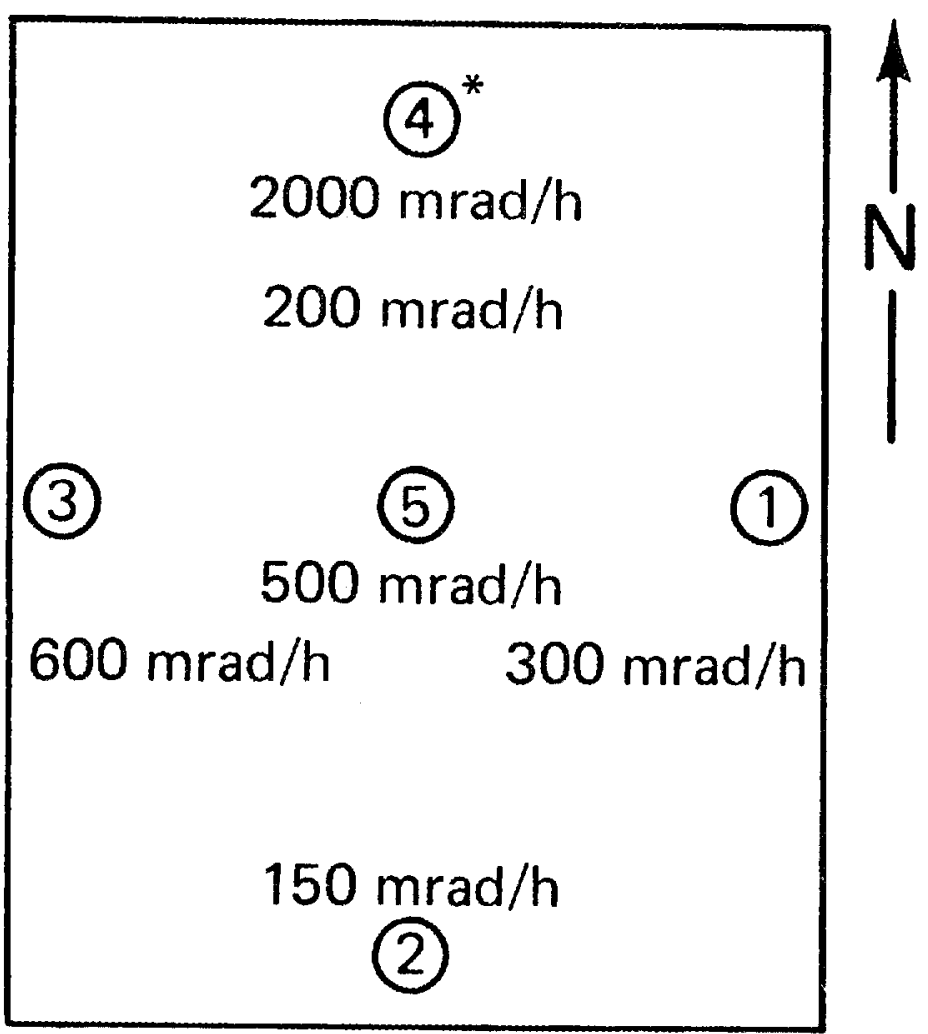

*SMEAR SAMPLE LOCATION

\section{SMEARABLE ACTIVITY PER $100 \mathrm{~cm}^{2}$}

\begin{tabular}{ccc}
\hline LOCATION & BETA-GAMMA & ALPHA \\
\hline 1 & $2 \mathrm{mrad} / \mathrm{h}$ & $<20 \mathrm{dpm}$ \\
2 & $4 \mathrm{mrad} / \mathrm{h}$ & $<20 \mathrm{dpm}$ \\
3 & $3 \mathrm{mrad} / \mathrm{h}$ & $<20 \mathrm{dpm}$ \\
4 & $3 \mathrm{mrad} / \mathrm{h}$ & $<20 \mathrm{dpm}$ \\
5 & $5 \mathrm{mrad} / \mathrm{h}$ & $<20 \mathrm{dpm}$
\end{tabular}

Fig. 6. Radiological survey results of the pump cell ( $1 \mathrm{mrad}=\mu \mathrm{Gy})$. Direct beta-gamma readings are marked. 
ORNL-DWG 84-12878

WELL CELL

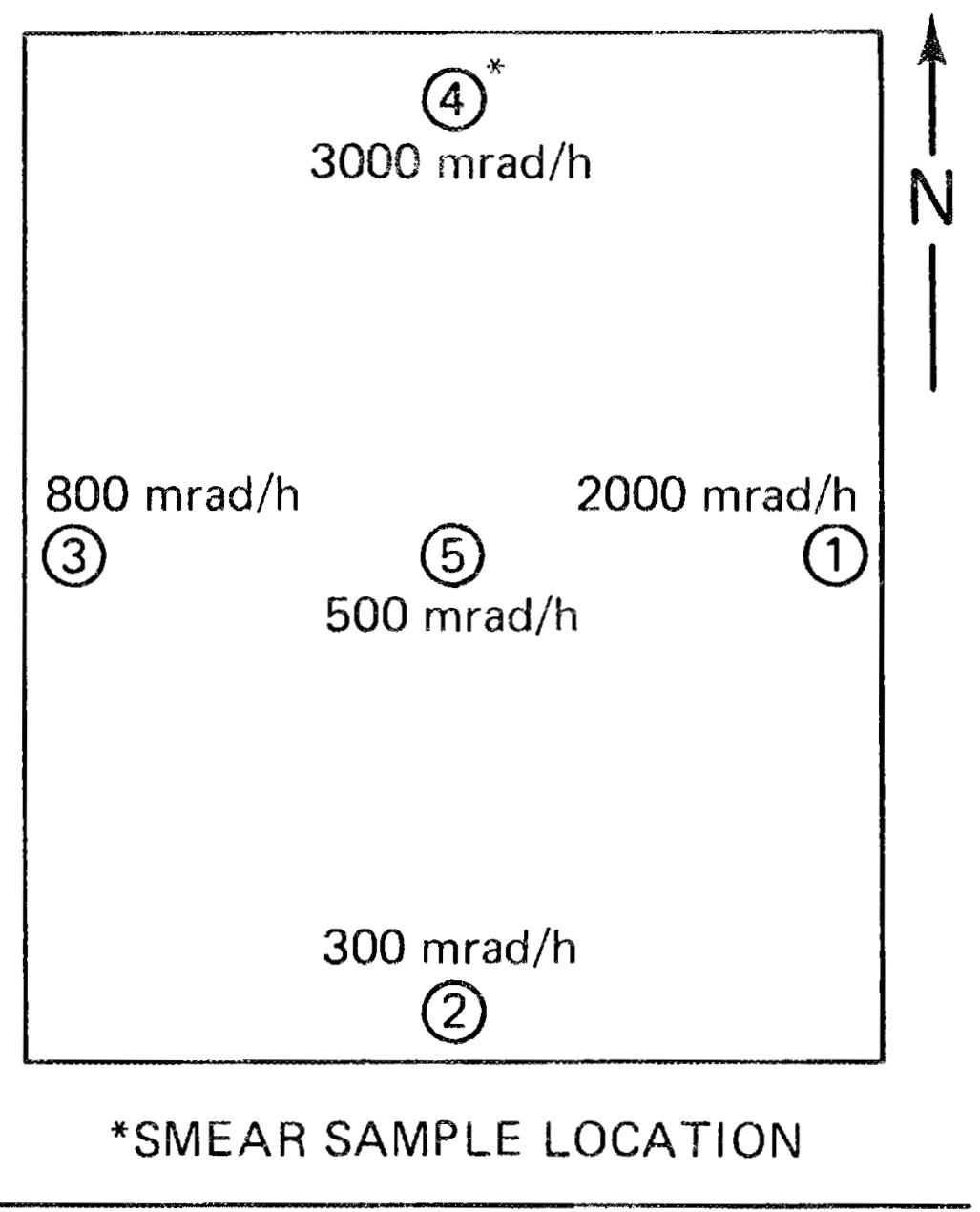

SMEARABLE ACTIVITY PER $100 \mathrm{~cm}^{2}$

\begin{tabular}{ccc}
\hline LOCATION & BETA-GAMMA & ALPHA \\
\hline 1 & $35 \mathrm{mrad} / \mathrm{h}$ & $<20 \mathrm{dpm}$ \\
2 & $35 \mathrm{mrad} / \mathrm{h}$ & $<20 \mathrm{dpm}$ \\
3 & $15 \mathrm{mrad} / \mathrm{h}$ & $<20 \mathrm{dpm}$ \\
4 & $20 \mathrm{mrad} / \mathrm{h}$ & $<20 \mathrm{dpm}$ \\
5 & $10 \mathrm{mrad} / \mathrm{h}$ & $<20 \mathrm{dpm}$
\end{tabular}

Fig. 1. Radiological survey results of the well cell (1 mrad $=10 \mu G y)$. Direct beta-gamma readings are marked. 
ORNL-DWG 84-12876

\section{ENGINE PAD}

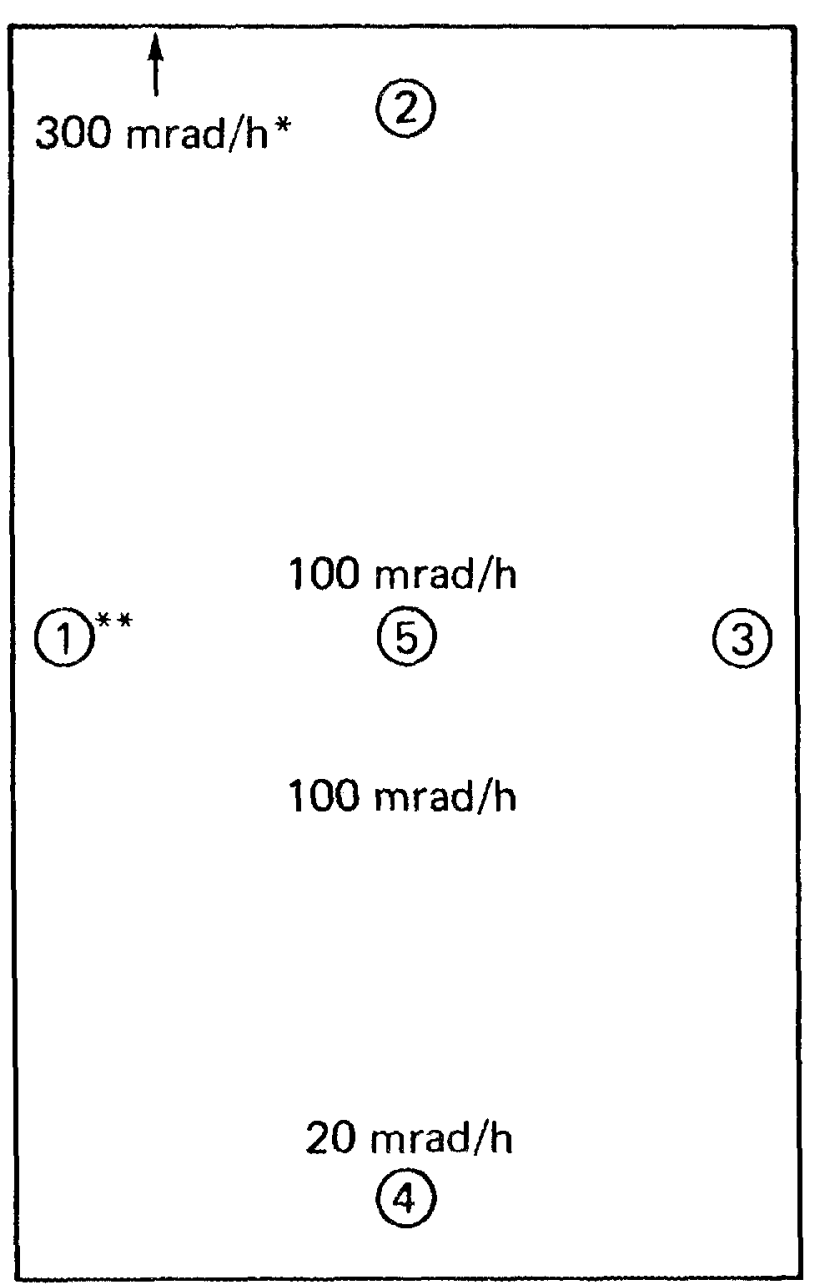

* READING AGAINST THE WALL * *MeAR SAMPLE LOCATION

SMEARABLE ACTIVITY PER $100 \mathrm{~cm}^{2}$

\begin{tabular}{ccr}
\hline LOCATION & BETA-GAMMA & ALPHA \\
\hline & & \\
1 & $0.75 \mathrm{mrad} / \mathrm{h}$ & $<20 \mathrm{dpm}$ \\
2 & $0.5 \mathrm{mrad} / \mathrm{h}$ & $<20 \mathrm{dpm}$ \\
3 & $1.0 \mathrm{mrad} / \mathrm{h}$ & $27 \mathrm{dpm}$ \\
4 & $1.0 \mathrm{mrad} / \mathrm{h}$ & $<20 \mathrm{dpm}$ \\
5 & $1.0 \mathrm{mrad} / \mathrm{h}$ & $<20 \mathrm{dpm}$
\end{tabular}

Fig. 8. Radiological survey results of the engine pad (1 $\mathrm{mrad}=10 \mu \mathrm{Gy})$. Smear sample locations and direct beta-gamna dose rates are marked. 


\subsubsection{Pump House}

The interior surfaces of the pump house were contaminated with fixed and removable activity from 0.2 to $80 \mathrm{mGy} / \mathrm{h}(20$ to $8000 \mathrm{mrad} / \mathrm{h}$ ) and 5 to $150 \mu \mathrm{Gy} / \mathrm{h}(0.5$ to $15 \mathrm{mrad} / \mathrm{h})$, respectively (Fig. 9). A radiation reading of $50 \mu G y / h$ ( 5 mrad/h) was ineasured directly above the sheet metal covering the concrete blocks of the valve pit. The interior of the pit was considered likely to be contaminated with fixed and removable activity.

\subsubsection{Soils}

S. F. Huang (EOSD, personal communication, September 1984) made direct beta-gamma readings on 6 - by 6 -m grids covering a 96 - by 60-in area that encompassed the OHF (see Fig. 10 for readings in excess of $3 \mu G y$ at 1 to $3 \mathrm{~cm}$ above the soil surface). Also illustrated in Fig. 10 are the locations of 17 deep soil cores taken near potential radiation hazards. Sections of these soil cores, of varying soil depths, were counted on a $15-\times 15-\mathrm{cm} \mathrm{NaI(TI)} \mathrm{detector} \mathrm{for} 5 \mathrm{~min}$, from which the gamma-ray spectrum (and total integral counts) over the energy range of 100 to $1500 \mathrm{keV}$ was obtained. The activity in $\mathrm{cps} / \mathrm{kg}$ is presented in rable 2 .

\subsubsection{Waste Pits}

In January of $1984 \mathrm{~S}$. F. Huang (EOSO, personal communication, September 1984) measured the absorbed dose rates under the roof of the waste pit ranging from 0.1 to $0.4 \mathrm{mgy} / \mathrm{h}(10$ to $40 \mathrm{mrad} / \mathrm{h})$. In the south pit, $45 \mathrm{~cm}$ of water covered a $10-\mathrm{cm}$ layer of sediment. In the north pit, approximately twice as much water $(90 \mathrm{~cm})$ covered a $10-\mathrm{cm}$ 


\section{PUMP ROOM}

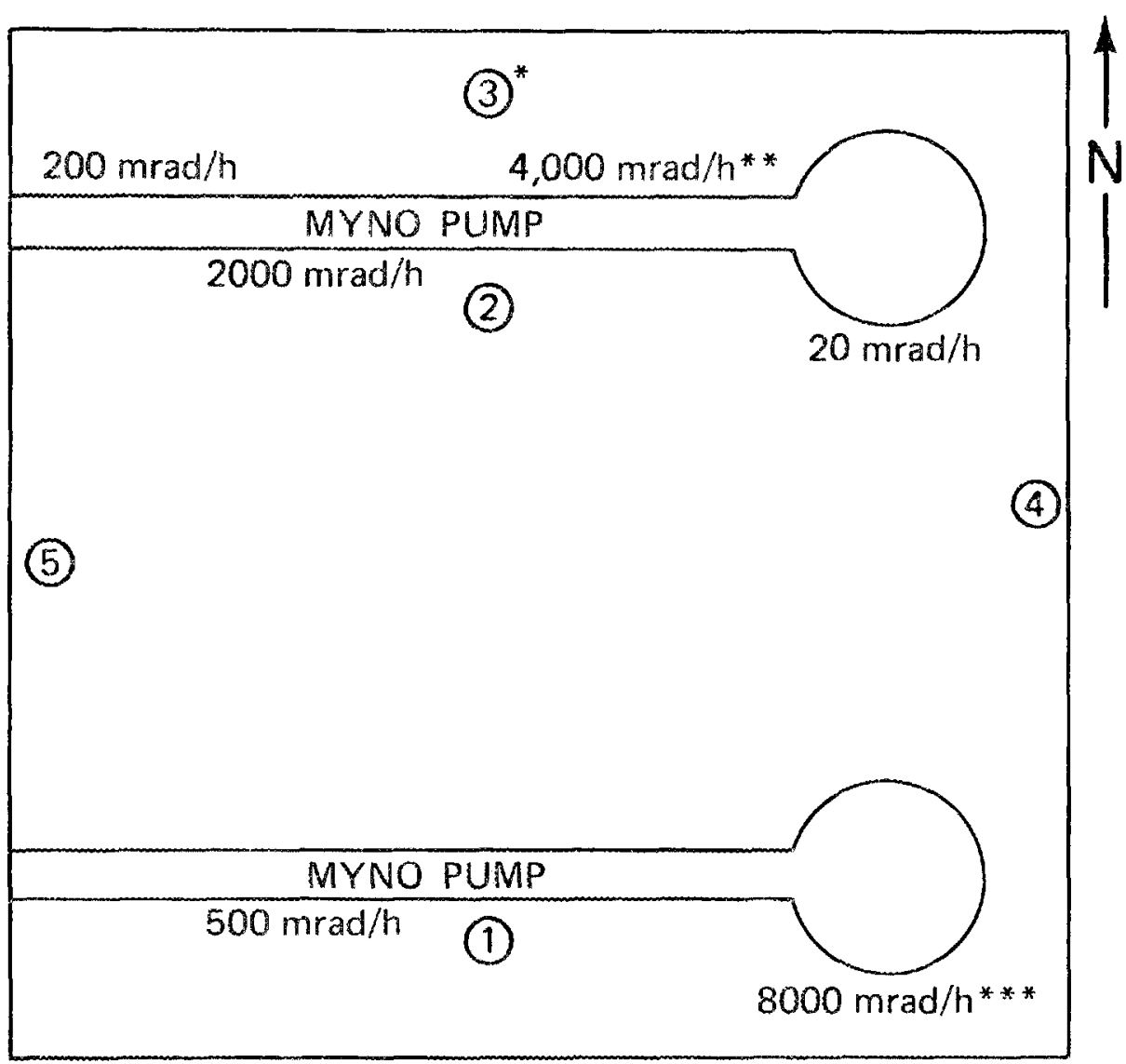

* SMEAR SAMPLE LOCATION

* READING AGAINST THE PUMP

** READING FROM UNDERNEATH A LEAD SHIELD

\begin{tabular}{crr}
\hline & \multicolumn{2}{c}{$\begin{array}{c}\text { SMEARABLE ACTIVITY } \\
\text { PER 100 cm }\end{array}$} \\
\hline LOCATION & BETA-GAMMA & ALPHA \\
\hline & & \\
1 & $0.5 \mathrm{mrad} / \mathrm{h}$ & $<20 \mathrm{dpm}$ \\
2 & $15.0 \mathrm{mrad} / \mathrm{h}$ & $<20 \mathrm{dpm}$ \\
3 & $10.0 \mathrm{mrad} / \mathrm{h}$ & $<20 \mathrm{dpm}$ \\
4 & $10.0 \mathrm{mrad} / \mathrm{h}$ & $<20 \mathrm{dpm}$ \\
5 & $8.0 \mathrm{mrad} / \mathrm{h}$ & $<20 \mathrm{dpm}$
\end{tabular}

Fig. 9. Radiological survey results of the pump room (1 mrad $=10 \mu G y)$. Direct beta gamma readings are marked. 


\section{SITE OF OLD HYDROFRACTURE FACILITY}

\section{- - UNDERGROUND WASTE TRANSFER LINES \\ (1) CORE - SITE 1 \\ - water AND SEDIMENT SAMPLES}

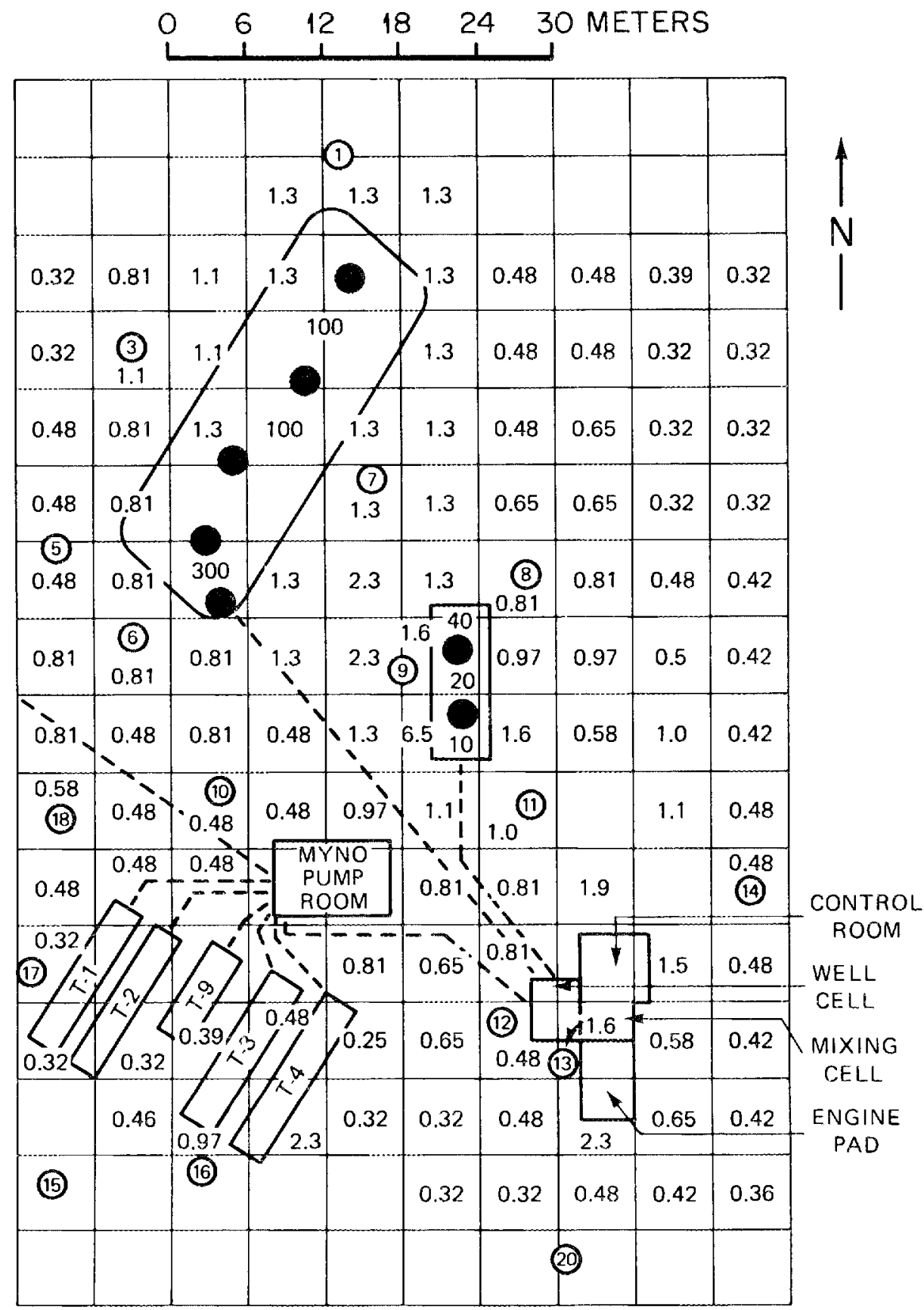

Fig. 10. Beta-ganma direct readings exceeding $0.3 \mathrm{mrad} / \mathrm{h}$ ( $1 \mathrm{mrad}=10 \mu \mathrm{Gy}$ ) at 1 to $3 \mathrm{~cm}$ above the surface. 
Tab7e 2. Gamma screening of deep soil cores

Deep soil core sites

\begin{tabular}{|c|c|c|c|c|c|c|c|c|c|c|c|c|c|c|c|c|c|}
\hline & & & & & & & $e p$ & 11 & 5 & & & & & & & & \\
\hline & 1 & 3 & 5 & 6 & 7 & 8 & 9 & 10 & 11 & 12 & 13 & 14 & 15 & 16 & 17 & 18 & 20 \\
\hline $\begin{array}{l}\text { Soll depth } \\
\text { in meters }\end{array}$ & $\cdots$ & $\cdots$ & $\cdots$ & $\cdots$ & $\cdots$ & & $\cdots$ & $\langle c|$ & $\mathrm{kg})^{\mathrm{a}}$ & - & 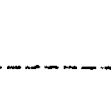 & & $\cdots-$ & & & - & - \\
\hline $\begin{array}{l}0.0-0.3 \\
0.3-0.6 \\
0.6-1.2 \\
1.2-1.8 \\
1.8-2.4 \\
2.4-3.1 \\
3.1-3.7 \\
3.7-4.3 \\
4.3-4.9 \\
4.9-5.5\end{array}$ & $\begin{array}{r}<80 \\
33 \\
44 \\
44 \\
34 \\
42 \\
\text { NS } \\
\text { NS } \\
\text { NS } \\
\text { NS }\end{array}$ & $\begin{array}{r}66 \\
29 \\
55 \\
420 \\
65 \\
45 \\
32 \\
34 \\
\text { NS } \\
\text { NS }\end{array}$ & $\begin{array}{l}35 \\
41 \\
30 \\
28 \\
32 \\
33 \\
31 \\
\text { NS } \\
\text { NS } \\
\text { NS }\end{array}$ & $\begin{array}{l}47 \\
32 \\
34 \\
37 \\
29 \\
45 \\
46 \\
\text { NS } \\
\text { NS } \\
\text { NS }\end{array}$ & $\begin{array}{l}96 \\
38 \\
38 \\
31 \\
32 \\
34 \\
34 \\
\text { NS } \\
\text { NS } \\
\text { NS }\end{array}$ & $\begin{array}{l}\text { NS } \\
\text { NS } \\
44 \\
24 \\
34 \\
28 \\
53 \\
26 \\
37 \\
35\end{array}$ & $\begin{array}{r}<30 \\
28 \\
60 \\
31 \\
37 \\
35 \\
37 \\
35 \\
28 \\
\text { NS }\end{array}$ & $\begin{array}{r}470 \\
120 \\
240 \\
34 \\
38 \\
25 \\
32 \\
38 \\
66 \\
46\end{array}$ & $\begin{array}{l}N S^{b} \\
N S \\
35 \\
36 \\
38 \\
38 \\
34 \\
30 \\
30 \\
29\end{array}$ & $\begin{array}{r}\text { NS } \\
\text { NS } \\
36 \\
39 \\
34 \\
34 \\
81 \\
240 \\
\text { NS } \\
\text { NS }\end{array}$ & $\begin{array}{r}\text { NS } \\
\text { NS } \\
130 \\
68 \\
70 \\
46 \\
69 \\
260 \\
40 \\
50\end{array}$ & $\begin{array}{l}\text { NS } \\
\text { NS } \\
81 \\
84 \\
43 \\
45 \\
37 \\
47 \\
34 \\
53\end{array}$ & $\begin{array}{l}51 \\
43 \\
40 \\
33 \\
40 \\
31 \\
64 \\
41 \\
42 \\
35\end{array}$ & $\begin{array}{l}88 \\
43 \\
36 \\
42 \\
53 \\
54 \\
\text { NS } \\
33 \\
32 \\
67\end{array}$ & $\begin{array}{l}29 \\
29 \\
30 \\
32 \\
40 \\
\text { NS } \\
\text { NS } \\
\text { NS } \\
\text { NS } \\
\text { NS }\end{array}$ & $\begin{array}{l}43 \\
32 \\
41 \\
33 \\
30 \\
34 \\
31 \\
31 \\
34 \\
\text { NS }\end{array}$ & $\begin{array}{r}<30 \\
20 \\
27 \\
36 \\
31 \\
31 \\
37 \\
39 \\
41 \\
\text { NS }\end{array}$ \\
\hline $\begin{array}{l}\text { Mean } \\
\text { concentration }\end{array}$ & ns 39 & 93 & 33 & 38 & 43 & 35 & 36 & 111 & 34 & 77 & 92 & 52 & 42 & 50 & 32 & 34 & 33 \\
\hline $\begin{array}{l}\text { Coordinates } \\
\text { in meters }\end{array}$ & & & & & & & & & & & & & & & & & \\
\hline $\begin{array}{l}\text { North } \\
\text { East }\end{array}$ & $\begin{array}{l}5298 \\
8708\end{array}$ & $\begin{array}{l}5273 \\
8690\end{array}$ & $\begin{array}{l}5262 \\
8682\end{array}$ & $\begin{array}{l}5256 \\
8690\end{array}$ & $\begin{array}{l}5268 \\
8711\end{array}$ & $\begin{array}{l}5262 \\
8717\end{array}$ & $\begin{array}{l}5255 \\
8708\end{array}$ & $\begin{array}{l}5246 \\
8697\end{array}$ & $\begin{array}{l}5244 \\
8720\end{array}$ & $\begin{array}{l}5229 \\
8717\end{array}$ & $\begin{array}{l}5225 \\
8722\end{array}$ & $\begin{array}{l}5236 \\
8737\end{array}$ & $\begin{array}{l}5223 \\
8681\end{array}$ & $\begin{array}{l}5217 \\
8697\end{array}$ & $\begin{array}{l}5232 \\
8678\end{array}$ & $\begin{array}{l}5246 \\
8623\end{array}$ & $\begin{array}{l}5202 \\
8723\end{array}$ \\
\hline
\end{tabular}

aGamma activity is in counts per second per kilogram of moist soil (cps/kg). Natural background was approximate $1 \mathrm{y} 20$ to $40 \mathrm{cps} / \mathrm{kg}$ on the $15-$ by $15-\mathrm{cm} \mathrm{NaI}$ (TI) detector over the energy span of 100 to $1500 \mathrm{keV}$ (S. F. Huang, EOSO, personal communication, September 1984).

$b_{N S}=$ not sampied. 
Table 3. Waste pit inventories of radionuclides ${ }^{a}$

\begin{tabular}{|c|c|c|c|c|c|}
\hline & $\begin{array}{l}\text { Average depth } \\
\text { and volume }\end{array}$ & $\begin{array}{l}\text { Radio- } \\
\text { nuclide }\end{array}$ & $\begin{array}{c}\text { Concentration } \\
(\mathrm{Bq} / \mathrm{mL} \text { or } \\
\mathrm{Bq} / \mathrm{g})\end{array}$ & \multicolumn{2}{|c|}{ Inventory } \\
\hline $\begin{array}{c}\text { South cell } \\
\text { water }\end{array}$ & $\begin{array}{r}45 \mathrm{~cm} \\
6.3 \times 10^{3} \mathrm{~L}\end{array}$ & $\begin{array}{l}135 \mathrm{Cs} \\
239 \mathrm{Pu} \\
238 \mathrm{Pu} \\
241 \mathrm{Am} \\
244 \mathrm{Cm} \\
90 \mathrm{Sr}\end{array}$ & $\begin{array}{l}1.2 \times 10^{2} \\
1.1 \\
2.2 \\
1.0 \\
1.0 \\
15.0\end{array}$ & $\begin{array}{l}0.8 \\
0.007 \\
0.01 \\
0.006 \\
0.006 \\
0.09\end{array}$ & $\begin{array}{r}20.0 \\
0.2 \\
0.4 \\
0.2 \\
0.2 \\
3.0\end{array}$ \\
\hline $\begin{array}{r}\text { South cell } \\
\text { sediment }\end{array}$ & $\begin{array}{l}10 \mathrm{~cm} \\
1.4 \times 10^{3} \mathrm{~L}\end{array}$ & $\begin{array}{r}137 \mathrm{Cs} \\
60 \mathrm{Co} \\
239 \mathrm{Pu} \\
238 \mathrm{Pu} \\
241 \mathrm{ArI} \\
244 \mathrm{Cm} \\
90 \mathrm{Sr}\end{array}$ & $\begin{array}{l}4.0 \times 10^{3} \\
31.0 \\
97.0 \\
30.0 \\
17.0 \\
54.0 \\
7.8 \times 10^{2}\end{array}$ & $\begin{array}{l}6.0 \\
0.04 \\
0.1 \\
0.04 \\
0.02 \\
0.08 \\
1.0\end{array}$ & $\begin{array}{r}200.0 \\
1.0 \\
4.0 \\
1.0 \\
0.6 \\
2.0 \\
30.0\end{array}$ \\
\hline $\begin{array}{c}\text { North cell } \\
\text { water }\end{array}$ & $\begin{array}{l}90 \mathrm{~cm} \\
1.3 \times 10^{4} !\end{array}$ & $\begin{array}{l}137 \mathrm{Cs} \\
239 \mathrm{Pu} \\
238 \mathrm{Pu} \\
241 \mathrm{Am} \\
244 \mathrm{Cm} \\
90 \mathrm{Sr}\end{array}$ & $\begin{array}{l}2.1 \times 10^{2} \\
1.0 \\
1.5 \\
1.0 \\
1.0 \\
16.0\end{array}$ & $\begin{array}{l}3.0 \\
0.01 \\
0.02 \\
0.01 \\
0.01 \\
0.2\end{array}$ & $\begin{array}{r}80.0 \\
0.4 \\
0.5 \\
0.4 \\
0.4 \\
6.0\end{array}$ \\
\hline $\begin{array}{l}\text { North cell } \\
\text { sediment }\end{array}$ & $\begin{array}{l}10 \mathrm{~cm} \\
1.4 \times 10^{3} \mathrm{~L}\end{array}$ & $\begin{array}{r}137 \mathrm{Cs} \\
60 \mathrm{Co} \\
239 \mathrm{Pu} \\
238 \mathrm{Pu} \\
241 \mathrm{Am} \\
244 \mathrm{Cm} \\
90 \mathrm{Sr}\end{array}$ & $\begin{array}{r}3.1 \times 10^{3} \\
14.0 \\
42.0 \\
17.0 \\
13.0 \\
6.0 \times 10^{2} \\
4.0 \times 10^{2}\end{array}$ & $\begin{array}{l}4.0 \\
0.02 \\
0.06 \\
0.02 \\
0.02 \\
0.8 \\
0.6\end{array}$ & $\begin{array}{r}100.0 \\
0.6 \\
2.0 \\
0.6 \\
0.6 \\
20.0 \\
20.0\end{array}$ \\
\hline
\end{tabular}

a Taken from S. F. Huang (EOSO, personal communication, September 1984).

${ }^{b}$ Inventory in the sediment was estimated by assuming that the total volume was equal to the total dry weight. 
layer of sediment. The total radioactivity in the water and sediment was estimated to be $5 \mathrm{GBq}(0.7 \mathrm{Ci})$ and $10 \mathrm{GBq}(0.3 \mathrm{Ci})$. respectively The concentrations and inventories of the various radionuclides measured in the water and sediment taken from these pits are listed in Table 3.

\subsubsection{Waste Tanks}

S. F. Huang (EOSO, personal communication, September 1984) estimated the radioactivity in each tank to be between 22 and $37 \mathrm{TBq}$ (600 to $1000 \mathrm{Ci})$. The total quantity of sludge in the tanks was estimated to be

$2 \times 10^{6} \mathrm{~L}$, containing on the order of $170 \mathrm{rBq}(4.6 \mathrm{kCi})$. An estimate of the activity in each of the tanks, and the assumptions used in making the estimates are presented in Table 4. Water taken from the "dry wells" (monitoring wells located within the concrete enclosure built to contain each of the tanks) have shown slightly elevated levels of beta activity (maximum of $1.2 \mathrm{~Bq} / \mathrm{mL}$ ). Also, the soil core taken directly to the southwest of tank T-4 (soil core 16) showed some elevated levels of gamma activity at soil depths of approximately $5 \mathrm{~m}$ (see Table 2). The possibility (or likelihood) has to be taken into consideration that the carbon steel tanks, which are inclined to rust (a) though cathodically protected), may have developed leaks over the years.

\subsubsection{Waste Impoundment}

\subsubsection{Pond Water}

Pond water from the OHF impoundment was sampled in May of 1985 by Stansfield and Francis (1986). The sample was taken from the center of the impoundment when the depth of water was approximately $1 \mathrm{~m}(3 \mathrm{ft})$. The sample, which was not filtered, was analyzed for heavy metals, 
Table 4. Estimate of residual radioactivity in waste tanks at the old hycrofracture facility

\begin{tabular}{|c|c|c|c|c|c|c|}
\hline \multirow[t]{2}{*}{ Tank } & \multirow{2}{*}{$\begin{array}{c}\text { Tank } \\
\text { capacity } \\
\text { (L) }\end{array}$} & \multirow{2}{*}{$\begin{array}{l}\text { Tank } \\
\text { diameter } \\
(m)\end{array}$} & \multirow{2}{*}{$\begin{array}{c}\text { Tank } \\
\text { Jength } \\
\text { (m) }\end{array}$} & \multirow{2}{*}{$\begin{array}{l}\text { Sludge } \\
\text { volume } \\
\text { (L) }\end{array}$} & \multicolumn{2}{|c|}{$\begin{array}{c}\text { Sludge } \\
\text { activities }\end{array}$} \\
\hline & & & & & $\overline{(T B q)}$ & $(\mathrm{KCi})$ \\
\hline$r-1$ & $5.9 \times 10^{4}$ & 2.4 & 13.0 & $4.2 \times 10^{3}$ & 40 & 1.0 \\
\hline$T-2$ & $5.9 \times 10^{4}$ & 2.4 & 13.0 & $4.2 \times 10^{3}$ & 40 & 1.0 \\
\hline$T-9$ & $4.4 \times 10^{4}$ & 3.7 & 5.9 & $2.2 \times 10^{3}$ & 30 & 0.6 \\
\hline$T-3$ & $1.1 \times 10^{5}$ & 3.2 & 13.0 & $4.9 \times 10^{3}$ & 40 & 1.0 \\
\hline$T-4$ & $1.1 \times 10^{5}$ & 3.2 & 13.0 & $4.9 \times 10^{3}$ & 40 & 1.0 \\
\hline
\end{tabular}

afrom S. F. Huang (EOSD, personal communication, September 1984). The estimate was based on the assumption of $30 \mathrm{~cm}$ thickness of residual sludge (a rough estimate from operational experience) at a concentration of $0.26 \mathrm{Ci} / \mathrm{L}$. 
Table 5. Concentration and inventory of nonradioactive contaminants in OHF pond watera

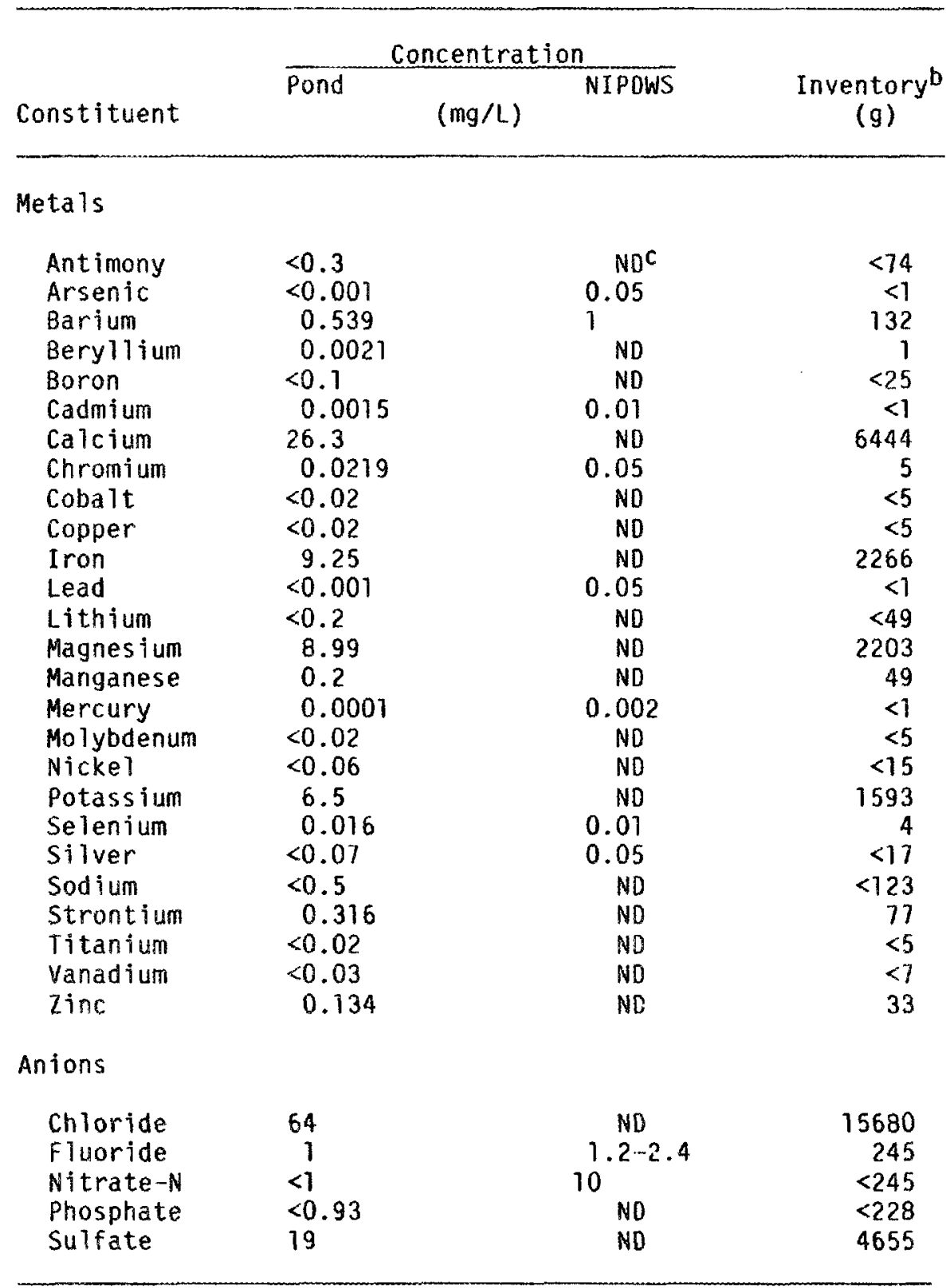

a Data taken from $R, G$. Stansfield and C.W. Francis, 1986. Characterization of the 0ld Hydrofracture Facility (OHF) Impoundment, ORNL/TM 9990, Oak Ridge National Laboratory. Oak Ridge, Tennessee.

D Inventory based on pond water volume of $2.45 \times 10^{5} \mathrm{~L}$.

$C_{\text {ND }}=$ level not defined by NIPOWS (National Interim Primary Drinking Water Standards). 
Table 6. Concentration and inventory of organic chemicals in OHF pond watera

\begin{tabular}{|c|c|c|c|}
\hline \multirow[b]{2}{*}{ Constituent } & \multicolumn{2}{|c|}{ Concentration } & \multirow[b]{2}{*}{$\begin{array}{c}\text { Inventory } \\
(g)\end{array}$} \\
\hline & Pond & NIPOWS & \\
\hline \multicolumn{4}{|c|}{ Herbicides/pesticides } \\
\hline $\begin{array}{l}\text { Endrin } \\
\text { Lindane } \\
\text { Methoxychlor } \\
\text { Toxaphene }\end{array}$ & $\begin{array}{l}<0.0001 \\
<0.0001 \\
<0.0002 \\
<0.002\end{array}$ & $\begin{array}{l}0.002 \\
0.004 \\
0.1 \\
\quad{ }_{N D}{ }^{C}\end{array}$ & $\begin{array}{l}<0.1 \\
<0.1 \\
<0.1 \\
<0.5\end{array}$ \\
\hline \multicolumn{4}{|l|}{ Organic compounds ${ }^{d}$} \\
\hline $\begin{array}{l}\text { PCBs } \\
\text { Phenols } \\
\text { TOC } \\
\text { TOX }\end{array}$ & $\begin{array}{l}0.0001 \\
<0.0001 \\
16.5 \\
0.132\end{array}$ & $\begin{array}{l}\text { ND } \\
\text { ND } \\
\text { ND } \\
\text { ND }\end{array}$ & $\begin{array}{c}<0.1 \\
<0.1 \\
4043 \\
32\end{array}$ \\
\hline
\end{tabular}

a Data taken from R. G. Stansfield and C. W. Francis, 1986, Characterization of the 01d Hydrofracture Facility (OHF) Impoundment, ORNL/TM-9990, Oak Ridge National Laboratory, Oak Ridge, Tennessee.

${ }^{b}$ Inventory base on pond water volume of $2.45 \times 10^{5} \mathrm{~L}$.

${ }^{c_{N D}}=1$ imit not defined by NIPOWS (National Interim Primary Drinking water Standards).

\footnotetext{
$\mathrm{d}_{\mathrm{PCB}}=$ polychlorinated biphenyls, $T O C=$ total organic carbon, TOX = total organic halides.
} 
Table 7. Concentration and inventory of radionuclides in OHF pond water*

\begin{tabular}{|c|c|c|c|c|c|c|}
\hline \multirow[b]{2}{*}{ Radionuclide } & \multicolumn{3}{|c|}{ Stansfield and Francis $(1986)^{a}$} & \multicolumn{3}{|c|}{ S. F. Huang ${ }^{b}$} \\
\hline & $(B q / L)$ & $\begin{array}{c}\text { Inve } \\
(\mathrm{MBq})\end{array}$ & $\begin{array}{l}\text { tory } \\
\qquad(\mathrm{mC} i)\end{array}$ & $\begin{array}{c}\text { Concentration } \\
(\mathrm{Bq} / \mathrm{L})\end{array}$ & $\begin{array}{l}\text { Inv } \\
(M B q)\end{array}$ & $\begin{array}{l}\text { ory } \\
\text { (mCi) }\end{array}$ \\
\hline $\begin{array}{l}\text { Gross alpha } \\
\text { Gross beta } \\
\text { Americium-241 } \\
\text { Cesium-137 } \\
\text { Coba } 7 t-60 \\
\text { Curium-244 } \\
\text { Plutonium-238 } \\
\text { Plutonium-239 } \\
\text { Radium-226 } \\
\text { Strontium-90 } \\
\text { Uranium-234 } \\
\text { Uranium-238 }\end{array}$ & $\begin{array}{c}11 \\
9400 \\
0.24 \\
3900 \\
27 \\
6.8 \\
0.17 \\
0.052 \\
0.015 \\
4400 \\
1.5 \\
0.375\end{array}$ & $\begin{array}{c}2.7 \\
2303 \\
0.1 \\
956 \\
6.6 \\
1.7 \\
<0.1 \\
<0.1 \\
<0.1 \\
1080 \\
0.4 \\
0.1\end{array}$ & $\begin{array}{l}0.07 \\
63 \\
<0.01 \\
26 \\
0.18 \\
0.05 \\
<0.01 \\
<0.01 \\
<0.01 \\
29 \\
0.01 \\
<0.01\end{array}$ & $\begin{array}{r}\text { NR } C \\
N R \\
N R \\
29000 \\
27 \\
N R \\
N R \\
\text { NR } \\
\text { NR } \\
7100 \\
\text { NR } \\
\text { NR }\end{array}$ & $\begin{array}{r}N R \\
N R \\
N R \\
2100 \\
2 \\
N R \\
N R \\
N R \\
N R \\
514 \\
N R \\
N R\end{array}$ & $\begin{array}{l}\text { NR } \\
\text { NR } \\
\text { NR } \\
57 \\
0.05 \\
\text { NR } \\
\text { NR } \\
\text { NR } \\
\text { NR } \\
14 \\
\text { NR } \\
\text { NR }\end{array}$ \\
\hline
\end{tabular}

avalue used for pond volume, $2.45 \times 10^{5} \mathrm{~L}$ (R. G. Stansfield and C. W. Francis, 1986, Characterization of the 0ld Hydrofracture Facility (OHF) Impoundment, ORNL/TM-9990, Oak Ridge National Laboratory, Oak Ridge, Tennessee).

bvalue used for pond volume, $7.24 \times 10^{4} \mathrm{~L}$ (S. F. Huang, EOSO, personal communication, September 3984 ).

$C_{N R}=$ not reported. 
herbicides/pesticides, PCBs, and radionuclides (Tables 5, 6, and 7). The $\mathrm{pH}$ of the pond water was neutral (7.05) with specific conductance of $224 \mathrm{\mu S} / \mathrm{cm}$. The analyses indicated that arsenic, barium, cadmium, chromium, fluoride, mercury, lead, and nitrate, as well as the pesticides and herbicides, were below the maximum allowable NIPOWS (National Interim Primary Orinking water Standards). The measured concentration of selenium $(0.016 \mathrm{mg} / \mathrm{L})$, slightly over the NIPOWS level $(0.01 \mathrm{mg} / \mathrm{L})$, and the detection limit of silver $(0.07 \mathrm{mg} / \mathrm{L})$ determined in the pond water were the only indications that chemical constituents exceeded the allowable NIPOWS levels. Detectable concentrations of PCBs were observed in the pond water $(0.0001 \mathrm{mg} / \mathrm{L})$ and counts of coliforin bacteria ( 8 counts per $100 \mathrm{~mL}$ ) were in excess of the NIPOWS. The concentration of total organic halides (TOX) was $0.13 \mathrm{mg} / \mathrm{L}$, and the total organic carbon content (TOC) was $16 \mathrm{ing} / \mathrm{L}$. Other than the radionuclide concentrations, which are discussed below, the water quality of pond water from the impoundment (based on these chemical analyses) was surprisingly high.

The principal radionuclides measured in the pond water were cesium-137 and strontium-90 (Table 7). Stansfield and Francis (1986) observed similar concentrations of the two radionuclides [approximately $4000 \mathrm{~Bq} / \mathrm{L}$ (equivalent to $109 \mathrm{pCi} / \mathrm{mL}$ )], while S. F. Huang (EOSO, personal comnunication, September 1984). who sampled the impoundment in September 1983, observed approximately four times more cesium-137 than strontium 90 [29000 and $7100 \mathrm{~Bq} / \mathrm{L}$, respectively (equivalent to 800 and $193 \mathrm{p}(\mathrm{i} / \mathrm{mL})$. The concentrations of these two radionuclides measured by Huang et al. were two to seven times that measured by Stansfield and 
Francis (see Table 7). On the other hand, cobalt-60 concentrations in the pond water were identical. Stansfield and Francis (1986) also measured gross alpha and gross beta activity in the pond water. Gross beta activity was approximately twice the activity of strontiun-90. implying that the gross beta activity resulted largely from the decay of strontium-90 and yttrium-90. Gross alpha activity was less than $1 \%$ of the gross beta and cesium-137 activities combined. Curium-244 appeared to be the dominant transuranic radionuclide measured $(6.8 \mathrm{~Bq} / \mathrm{L}$ or $<0.2 \mathrm{p}(\mathrm{i} / \mathrm{mL})$. The volume of pond water retained in the impoundment was estimated by Stansfield and Francis to be approximately three times that estimated by S. F. Huang (EOSO, persona) communication, September 1984). The difference between time of the year in sampling, May for Stansfield and Francis and September for S. F. Huang (EOSO, personal communication, September 1984), is likely the major cause for the differences in estimates of pond water volumes. As a consequence, there was not a major difference in estimates of the radionuclide inventory between the two studies. For example, the total inventory of strontium-90 and cesium-137 (approximately $1 \mathrm{GBq}$ or $0.03 \mathrm{Ci}$ for each radionuclide) was not substantially different from the estimate by Huang et a1. (2.1 and $0.5 \mathrm{GBq}$, for cesium-137 and strontium-90, respective $7 y)$.

\subsubsection{Impoundment sediment}

S. F. Huang (EOSO, personal communication, September 1984) and Stansfield and Francis (1986) have measured concentrations of potential contaminants in the sediment of the oHF impoundment. The principal purpose of the latter study was to determine if the sediment would be classified as a hazardous waste under RCRA regulations. Thus, the 
emphasis in this study was centered more along the analyses of sediment for nonradioactive contaminants, whereas the earlier study was principally concerned about the radiological characteristics of the impoundment in support of decontamination and decommissioning (D\&D) activities. In the federal regulation 40 CFR 261 promulgated under RCRA (USEPA 1980), a solid waste is hazardous if it exhibits any of the defined characteristics of ignitability, corrosivity, reactivity, or extraction procedure (EP) toxicity. The EP toxicity is of primary concern because the inherent physical and chemical characteristics of the sediment rule out classification as a hazardous waste based on ignitability or reactivity. Since the pH of the sediment was 5.2 , the waste will not be classified as having the characteristic of corrosivity as defined under the regulations. The EP toxicity characteristic is based on measured concentrations of eight elements of the National Interim Primary Drinking Water Standard (NIPOWS) and six herbicides and pesticides in the filtrate of a 24-hr solid waste extraction test (USEPA 1982). If the levels of these constituents exceed established maximum permissible concentrations, then the waste is considered hazardous based on the characteristic of toxicity.

The sediment was thoroughly tested for its toxicity characteristics by replicate samplings and extractions of the sediment by the EP. For example, the sediment was tested for concentrations of metals in its EP extracts a total of six times (sediment collected from three sample locations at two dates). Analyses for the herbicides/pesticides in the extracts were conducted three times (sediment collected from three sample locations in the impoundment at a single sampling date). 

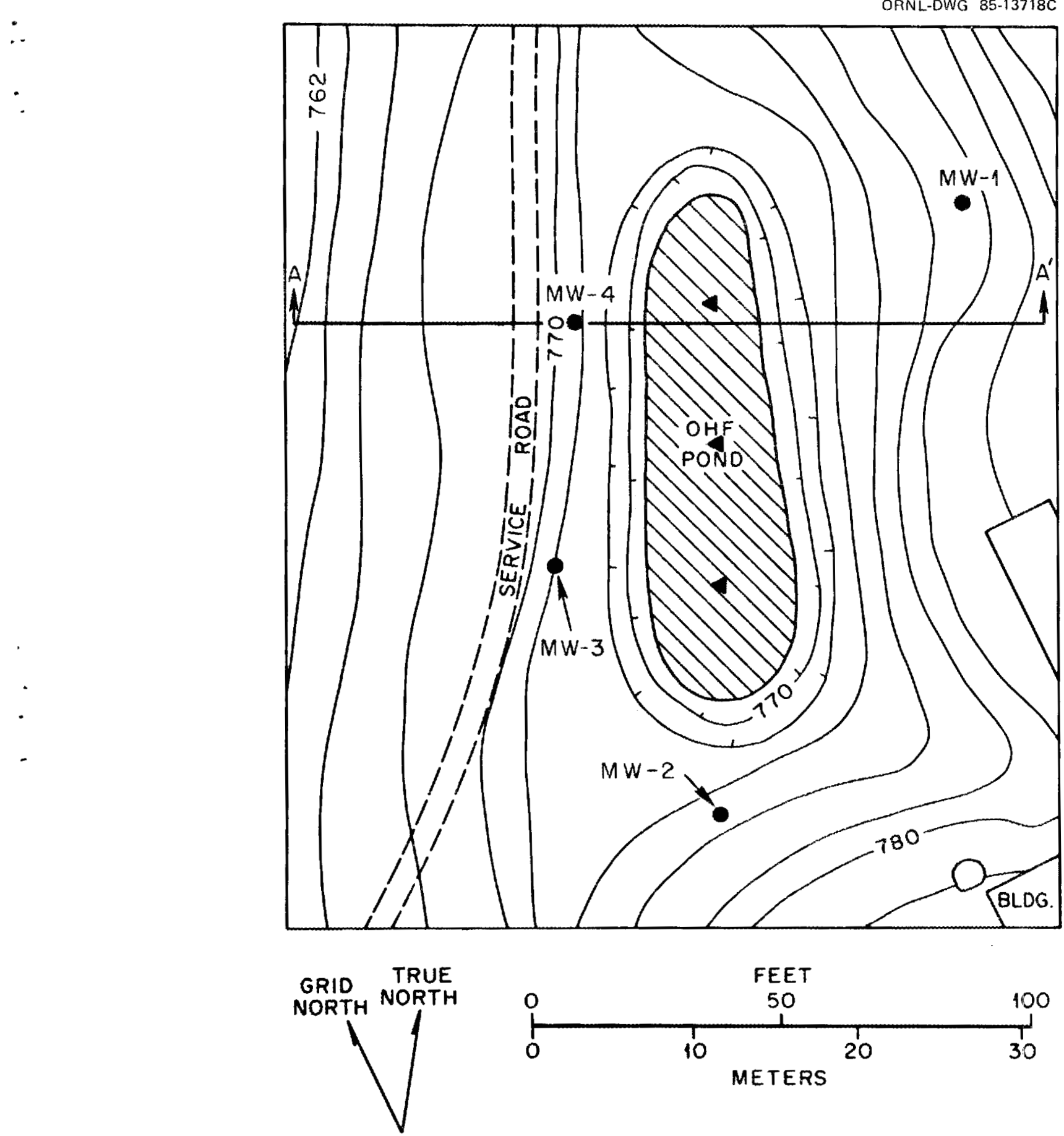

- MONITORING WELL

A-A' GEOLOGIC SECTION LINE

- sediment sampling location

Fig. 11. Location of sediment sampling sites and groundwater monitoring wells. 
Table 8. Concentration of RCRA regulated constituents in EP extracts of sediment from the OHF impoundment

\begin{tabular}{lcc}
\hline Constituent & $\begin{array}{c}\text { Maximum allowable } \\
\text { concentration } \\
(\mathrm{mg} / \mathrm{L})\end{array}$ & $\begin{array}{c}\text { Measured } \\
\text { concentrationa } \\
(\mathrm{mg} / \mathrm{L})\end{array}$ \\
\hline Arsenic & 5 & $<0.60$ \\
Barium & 100 & 0.307 \\
Cadinium & 1 & 0.0092 \\
Chromium & 5 & 0.0492 \\
Lead & 5 & $<0.0232$ \\
Mercury & 0.2 & 0.065 \\
Selenium & 1 & $<1.2$ \\
Silver & 5 & $<0.2$ \\
Endrin & 0.02 & $<0.0001$ \\
Lindane & 0.04 & 0.0001 \\
Methoxychlor & 10 & $<0.0002$ \\
Toxaphene & 0.5 & $<0.002$ \\
2,4-D & 10 & $<0.005$ \\
2,4,5-DTP & 1 & $<0.005$ \\
& &
\end{tabular}

a Taken from R. G. Stansfield and C.W. Francis, 1986, Characterization of the 0ld Hydrofracture Facility (OHF) Impoundment, ORNL/TM-9990, Oak Ridge National Laboratory, Oak Ridge, Tennessee. Means concentrations from six replicated EP extracts for metals (sediment from three sample locations at two dates). For the herbicides/pesticides, the concentrations are mean concentrations from three replicated EP extracts (sediment from three sample locations at a single date). 
Sediment sampling locations are illustrated in Fig. 11. Sampling was conducted in November 1984 and February 1985. A summary of these measurements are presented in Table 8.

Where analytical detection limits were low enough, the concentrations of the RCRA regulated constituents measured in the EP extracts were well below the RCRA maximum allowable concentrations. Selenium concentrations in the EP extracts of the sediment samples collected in November 1984 were determined by inductively coupled plasma (ICP) spectroscopy. For these samples, the detection 1 imit was $2.4 \mathrm{mg} / \mathrm{L}$, $1.4 \mathrm{mg} / \mathrm{L}$ in excess of the RCRA limit. The EP extracts of sediment samples taken in February 1985 were analyzed for selenium by atomic absorption spectroscopy (AA) in which the detection limit was 0.01 , well below the maximum allowable RCRA concentration. In all six EP extracts, the selenium concentrations were below detection by the particular analytical technique used. The mean of the two detection limits $(2.4$ and 0.01$)$ is $1.2 \mathrm{mg} / \mathrm{L}$. Thus, the mean selenium concentration reported in Table $8(1.2 \mathrm{mg} / \mathrm{L})$ is an artifact in reporting the detection limits of the analytical procedure, and should not imply that the concentrations of selenium in the EP extracts are in excess of the maximum allowable RCRA concentration.

Total concentrations of nonradioactive contaminants in sediment samples of the OHF impoundment were determined by both S. F. Huang (EOSO, personal communication, September 1984) and Stansfield and Francis (1986). The method of expressing their concentration, however, differed. Stansfield and Francis expressed the concentrations on the dry weight basis, while S. F. Huang (EOSO, personal communication, 
Table 9. Concentration and inventory of nonradioactive contaminants in sediment from the OHF impoundment

\begin{tabular}{|c|c|c|c|c|}
\hline \multirow[t]{2}{*}{ Contaninant } & \multicolumn{2}{|c|}{ Stansfield and Francis $(1986)^{a}$} & \multicolumn{2}{|c|}{ S. F. Huang } \\
\hline & $\begin{array}{c}\text { Concentration } \\
(\mathrm{mg} / \mathrm{kg})\end{array}$ & $\begin{array}{c}\text { Inventory } \\
(\mathrm{kg})\end{array}$ & $\begin{array}{c}\text { Concentration } \\
(\mathrm{mg} / \mathrm{kg})\end{array}$ & $\begin{array}{l}\text { Inventory } \\
(\mathrm{kg})\end{array}$ \\
\hline Antimony & $<194$ & $<3.69$ & $<1.0$ & $<0.08$ \\
\hline Arsenic & $<120$ & $<2.28$ & 1.1 & 0.09 \\
\hline Barium & 320 & 6.07 & $N R^{C}$ & NR \\
\hline Boron & 132 & 25 & NR & NR \\
\hline Cadinium & $<6.1$ & $<0.12$ & $<2.0$ & $<1.60$ \\
\hline Chromium & 342 & 6.50 & 17.0 & 1.30 \\
\hline Cobalt & 22.4 & 0.43 & NR & NR \\
\hline Copper & 140 & 2.66 & 18.0 & 1.40 \\
\hline Iron & 11200 & 403 & NR & NR \\
\hline Lead & $<156$ & $<2.97$ & 6.0 & 0.48 \\
\hline Manganese & 370 & 7.03 & NR & NR \\
\hline Mercury & NR & NR & $<2.3$ & $<0.18$ \\
\hline Molybdenum & $<15.5$ & $<0.29$ & NR & NR \\
\hline Nickel & 149 & 2.84 & NR & NR \\
\hline PCB & 2.9 & 0.05 & 3.5 & 2.80 \\
\hline Selenium & $<240$ & $<4.55$ & $<0.8$ & $<0.06$ \\
\hline Silver & $<40.9$ & $<0.78$ & NR & NR \\
\hline Vanadium & 79.1 & 1.50 & NR & NR \\
\hline Zinc & 162 & 3.07 & 16.0 & 1.20 \\
\hline
\end{tabular}

a Sediment concentrations by R. G. Stansfield and $C$. W. Francis [1986, Characterization of the Old Hydrofracture Facility (OHF) Impoundment, ORNL/TM-9990, Oak Ridge Nationa 1 Laboratory, Oak Ridge. Tennessee].

bSediment concentrations by S. F. Huang (EOSO, personal communication, September 1984) are on dry weight bas is and represent a mean of three sediment samples taken from the north, center, and south sections of the impoundment. Inventory calculated by stansfield and Francis was based on a total volume of sediment equal to $5.5 \times 10^{4} \mathrm{~L}$, a wet bulk density of $1.2 \mathrm{~kg} / \mathrm{L}$, and a moisture content of $71 \%$.

bsediment concentrations by Huang et al. are on a wet weight basis and represent a mean of five sediment samples taken from the center of the pond from a southwest to northeast direction. Inventory calculated by Huang et a 1 . was based on a total volume of sediment equal to $3.7 \times 10^{4} \mathrm{~L}$ and a wet bulk density of $2.16 \mathrm{~kg} / \mathrm{l}$.

$$
c_{N R}=\text { not reported } \text {. }
$$


September 1984) expressed the concentrations on a wet weight basis (Table 9). All things considered equal, the concentrations expressed on a dry weight basis should be higher than those on the same sediment on a wet weight basis. This is generally the case except for the case of PCBs in the sediment. Here, the concentrations deternined by $S$. F. Huang (EOSO, personal communication, September 1984) are higher than those determined by Stansfield and Francis. S. F. Huang (EOSO, persona) communication, September 1984) sampled the sediment in September 1983 , while Stansfield and Francis sampled in February 1985, which should not be a major factor assuming the degradation rates of PCBS to be relatively slow. Different volumes of sediment and bulk densities of the sediment were also used by each study to determine the total inventory. The basis of calculation for each of the studies is footnoted at the bottom of Table 9. Generally speaking, there was not a profound difference in the contaminant inventory between the two studies. The exception was the major difference in the estimated inventory of PCBs. S. F. Huang (EOSO, personal communication, September 1984) estimated the PCB inventory to be close to $3 \mathrm{~kg}$, while that calculated by stansfield and Francis was approximately 50 grams.

A similar trend in the concentrations of radionuclides in sediment taken frotn the OHF impoundment was noted between the two studies. For example, measurements by S. F. Huang (Eoso, personal communication, September 1984) were generally higher than those determined by Stansfield and Francis, even though S. F. Huang (EOSO, personal communication, September 1984) expressed the concentration on the wet weight bas is and Stansfield and Francis on a dry weight basis (see Table 10). The major 
Table 10. Concentration and inventory of radionuclides in sediment from the OHF impoundment

\begin{tabular}{|c|c|c|c|c|c|c|c|}
\hline \multirow[t]{3}{*}{ Radionuclide } & \multicolumn{3}{|c|}{ Stansfield and Francis $(1986)^{\mathrm{a}}$} & \multicolumn{4}{|c|}{ S. F. Huang ${ }^{b}$} \\
\hline & \multirow{2}{*}{$\begin{array}{c}\text { Concentration } \\
(\mathrm{Bq} / \mathrm{g})\end{array}$} & \multicolumn{2}{|c|}{ Inventory } & \multirow{2}{*}{\multicolumn{2}{|c|}{$\begin{array}{c}\text { Concentration } \\
(8 q / g)\end{array}$}} & \multicolumn{2}{|c|}{ Inventory } \\
\hline & & $(G B q)$ & (Ci) & & & $(\mathrm{GBq})$ & $(\mathrm{Ci})$ \\
\hline Gross alpha & 102 & 1.93 & 0.05 & $N R^{C}$ & & NR & NR \\
\hline Gross beta & 92700 & 1761 & 48 & NR & & NR & NR \\
\hline Cesium-134 & $<20$ & $<0.38$ & 0.01 & 100 & & 8.1 & 0.22 \\
\hline Cesium-137 & 125000 & 2370 & 64 & 180000 & 140 & & 388 \\
\hline Europium-154 & $<22.7$ & $<0.43$ & $<0.01$ & 390 & & 31 & 1 \\
\hline Uranium-234 & $<33.3$ & $<0.63$ & $<0.01$ & NR & & NR & NR \\
\hline Uranium-238 & $<612$ & $<11.6$ & $<1$ & 0.23 & & 0.0055 & $<0.01$ \\
\hline Americium-241 & $<20.0$ & $<0.38$ & $<0.01$ & 2.8 & & 0.22 & $<0.01$ \\
\hline Cobalt- -60 & 608 & 11.6 & 0.31 & 740 & & 50 & 1.6 \\
\hline Strontium-90 & 38800 & 737 & 20 & 9700 & & 10 & 21 \\
\hline
\end{tabular}

asediment concentrations by R. G. Stansfield and C. W. Francis, [1986, Characterization of the Old Hydrofracture Facility (OHF) Impoundment, ORNL/TM-9990, Dak Ridge Nationa 1 Laboratory, Oak Ridge, Tennessee] are on dry weight basis and represent a mean of three sediment samples taken from the north, center, and south sections of the impoundment. Inventory calculated by Stansfield and Francis was based on a total volume of sediment equal to $5.5 \times 10^{4} \mathrm{~L}$, a wet bulk density of $1.2 \mathrm{~kg} / \mathrm{L}$, and a moisture content of $71 \%$.

bSediment concentrations by S. F. Huang (EOSO, personal communication. September 1984) are on a wet weight bas is and represent a mean of five sediment samples (except the Europium-154 analys is which is from a single sediment sample) taken from the center of the pond from a southwest to northeast direction. Inventory calculated by Huang was based on a total volume of sediment equal to $3.7 \times 10^{4} \mathrm{~L}$ and a wet bulk density of $2.16 \mathrm{~kg} / \mathrm{L}$.

$$
c_{N R}=\text { not reported } \text {. }
$$


exception was the measurements for strontium-90. In this case, concentrations determined by $S$. F. Huang (EOSD, personal communication, Septenter 1984) were four times smaller than those determined by Stansfield and Francis. Ironically, the inventories of strontium-90 in the sediment by both studies were very close (737 and $770 \mathrm{GBa}$ or 20 and 21 (i). The basis for calculation of the inventories also differed with respect to volume of sediment, density, etc. (see footnotes at bottom of Table 10 for an explanation for the basis of calculation). As in the pond water, the bulk of the radioactivity is due to cesium-137 and strontium-90, and only a small but detectable fraction $(<0.05 \%)$ is transuranic related.

\subsubsection{Groundwater}

An indication that groundwater in the vicinity of the OHF is possibly contaminated are the strontium-90 measurements in samples of shallow groundwater taken immediately down-slope southeast of the OHF in the floodplain along Melton Branch (Spalding and Munro 1984). Strontium-90 concentrations (determined by a cherenkov counting technique) in groundwater samples taken in early spring of 1980 from shallow bore holes $(3.2 \mathrm{~cm}$ dian and $45 \mathrm{~cm}$ deep) were quite high (on the order of $10 \mathrm{kBq} / \mathrm{h}$ ) and were thought to be related to past operations of the facility. It was impossible to ascertain whether contamination resulted from subsurface contamination (e.g., from the migration of radionuclides from the waste impoundment or leaking waste storage tanks) or from surface contamination of this area by spillage of liquid waste during testing and injection processes. For example, in the late summer and fall of 1968 , two curies of strontium-90 was diverted 
"into a smal1 channel draining toward Melton Creek" from a test well approximately $6 \mathrm{~m}(20 \mathrm{ft})$ from Melton Branch that experienced considerable "bleed back" (unpublished January 7, 1969 correspondence to A. M. Weinberg from a committee to investigate the release). Test wells were used to investigate the grouting characteristics of certain injections. This particular test well was made following an injection by the hydrofracture unit into a fracture that contained approximately $130,000 \mathrm{~L}(35,000 \mathrm{gal})$ of residual water. Considerable pressure remained on the fracture, and a "bleed back" of contaminated water came up the test well, which was diverted to Melton Branch.

To determine the status of groundwater quality around the OHF impoundment, Stansfield and Francis (1986) installed four groundwater monitoring wells at the OHF site. One well was located to sample groundwater upgradient of the impoundment, and the other three were located to the west and south of the impoundment to sample groundwater downgradient to the impoundment (see fig. 11). The initial boring for the wells ranged in depth from 7.3 to $10.4 \mathrm{~m}(24$ to $34 \mathrm{ft})$. In each of the borings, monitoring wells were constructed of $3-i n(7.6-\mathrm{cm})$ diam fiberglass well screen and casing. A summary of the construction details and measured groundwater elevations, as we 11 as surveyed locations and elevation, is listed in Table 11.

Monitoring well $1(\mathrm{MW}-1)$ was located to provide a groundwater sampling point positioned upgradient (i.e., in the direction of increasing hydrostatic head of the groundwater table) of the impoundment. Its position, however, is only $27 \mathrm{~m}(95 \mathrm{ft})$ downgradient from the nearest waste trench in a formerly used low-level radioactive waste burial ground (SWSA 5, solid wastes storage area number 5). 
Table 11. Sumary of monitoring well location, construction data, and water levels at the OHF impoundment

\begin{tabular}{|c|c|c|c|c|}
\hline & $M W-1$ & $M W-2$ & $M W-3$ & $9 W-4$ \\
\hline $\begin{array}{l}\text { North grid } \\
\text { coordinate, ft }\end{array}$ & 17325.24 & 17235.06 & 17298.86 & 17339.13 \\
\hline $\begin{array}{l}\text { East grid } \\
\text { coordinate, ft }\end{array}$ & 28600.38 & 28504.80 & 28496.78 & 28519.01 \\
\hline $\begin{array}{l}\text { Top of well } \\
\text { casing elevation, } \mathrm{ft}\end{array}$ & 782.11 & 776.89 & 773.46 & 773.50 \\
\hline $\begin{array}{l}\text { Height of casing } \\
\text { above ground, ft }\end{array}$ & 2.80 & 2.70 & 2.90 & 2.90 \\
\hline $\begin{array}{l}\text { Ground surface } \\
\text { elevation, ft }\end{array}$ & 779.30 & 774.20 & 770.60 & 770.60 \\
\hline $\begin{array}{l}\text { Top of well } \\
\text { screen elevation, ft }\end{array}$ & 760.10 & 761.20 & 760.40 & 760.50 \\
\hline $\begin{array}{l}\text { Bottom of well } \\
\text { screen elevation, ft }\end{array}$ & 750.10 & 751.90 & 750.40 & 750.50 \\
\hline $\begin{array}{l}\text { Top of sand } \\
\text { pack elevation, ft }\end{array}$ & 769.80 & 768.20 & 764.60 & 762.60 \\
\hline $\begin{array}{l}\text { Bottom of we } 11 \\
\text { hole elevation, } \mathrm{ft}\end{array}$ & 744.30 & 750.20 & 746.60 & 746.60 \\
\hline $\begin{array}{l}\text { Diameter of well } \\
\text { pipe/screen, in. }\end{array}$ & 3.00 & 3.00 & 3.00 & 3.00 \\
\hline $\begin{array}{l}\text { Type material } \\
\text { of pipe/screen }\end{array}$ & $\begin{array}{l}\text { Fiber- } \\
\text { glass }\end{array}$ & $\begin{array}{l}\text { Fiber- } \\
\text { glass }\end{array}$ & $\begin{array}{l}\text { Fiber- } \\
\text { glass }\end{array}$ & $\begin{array}{l}\text { Fiber- } \\
\text { glass }\end{array}$ \\
\hline $\begin{array}{l}\text { Width of screen } \\
\text { opening, in. }\end{array}$ & 0.02 & 0.02 & 0.02 & 0.02 \\
\hline $\begin{array}{l}\text { Water level } \\
\text { elevation, ft, 4-8 -85 }\end{array}$ & 770.92 & 756.58 & 757.38 & 760.87 \\
\hline $\begin{array}{l}\text { Water level } \\
\text { elevation, } f t, 5-23-85\end{array}$ & 769.41 & 755.86 & 756.79 & 759.82 \\
\hline $\begin{array}{l}\text { Water level } \\
\text { elevation, ft, 6-4-85 }\end{array}$ & 758.92 & 755.37 & 756.14 & 759.36 \\
\hline $\begin{array}{l}\text { Water level } \\
\text { elevation, ft, 7-1-85 }\end{array}$ & 768.23 & 755.34 & 756.30 & 759.46 \\
\hline $\begin{array}{l}\text { Water level } \\
\text { elevation, } \mathrm{ft}, 7-30-85\end{array}$ & 768.04 & 755.47 & 756.50 & 759.66 \\
\hline
\end{tabular}

*Taken from R. G. Stansfield and C.W. Francis, 1986, Characterization of the 0ld Hydrofracture Facility (OHF) Impoundment, ORNL/TM-9990, Oak Ridge National Laboratory, Dak Ridge, Tennessee. 
The location of the other three monitoring wells were selected to determine if contaminants from the impoundment had migrated into the groundwater. The wells comply with the regulations promulgated in accordance with RCRA that specify that there be at least three hydraulically downgradient wells (i.e., in the direction of decreasing static head of the groundwater table). These downgradient wells were located at the boundary of the impoundment facility, which is described in the RCRA Permit Writer's Manual Groundwater Protection 40 CFR, Part 264, Subpart E, Draft (USEPA 1983) to be no more distant than the outside toe of any containment dike that may exist, plus $9.14 \mathrm{~m}(30 \mathrm{ft})$, for physically selecting an appropriate drill site.

The primary goal in analyzing groundwater samples taken from the monitoring wells was to determine whether the groundwater had been contaminated. To do this, samples were analyzed for those 30 constituents promulgated under RCRA regulations (USEPA 1980). The mean concentrations determined in groundwater sampled the first two quarters of 1985 from the three downgradient we11s are presented in 2 . The mean concentrations of all RCRA regulated constituents were below maximum allowable levels except those for gross alpha, gross beta, and coliform bacteria. Radioactivity as well as PCBs were detected in the upgradient well, indicating potential contamination by either leakage from the waste pits or from the many underground pipes in the OHF area. Many of the underground pipes lead to the waste pits located uphill and slightly to the southeast of the impoundment. Activity in this well could also have been caused by the leaching of radionuclides from wastes previously disposed of in the abandoned SWSA 5 burial 
ground. Counts of coliform bacteria in groundwater upgradient as well as downgradient were in excess of the NIPDWS. These counts may result from wildlife habitat such as waterfowl and terrestrial animals known to be in the area. Radioactivity was detected in all monitoring wells; however, well number 4 contained the highest concentration of cesium -137 and strontium-90. 
Table 12. Groundwater concentrations in wells downgradient from the OHF impoundment

\begin{tabular}{|c|c|c|}
\hline & $\begin{array}{l}\text { Maximum } \\
\text { level }\end{array}$ & $\begin{array}{c}\text { Measured } \\
\text { mean }\end{array}$ \\
\hline $\begin{array}{l}\text { National Interim Primary Drinking Water St } \\
\text { Arsenic } \\
\text { Barium } \\
\text { Cadmium } \\
\text { Chromium } \\
\text { Fluoride } \\
\text { Lead } \\
\text { Mercury } \\
\text { Nitrate-N } \\
\text { Selenium } \\
\text { Silver } \\
\text { Endrin } \\
\text { Lindane } \\
\text { Methoxychlor } \\
\text { Toxaphene } \\
2,4-0 \\
2,4,5-T p \text { Silvex } \\
\text { Radium-226, Bq/L } \\
\text { Gross alpha, Bq/L. } \\
\text { Gross beta, mR/yr } \\
\text { Coliform bacteria, counts per } 100 \mathrm{~mL} \text { ) }\end{array}$ & $\begin{array}{l}\text { andards } \\
0.05 \\
1 \\
0.01 \\
0.05 \\
1.4-2.4 \\
0.05 \\
0.002 \\
10 \\
0.01 \\
0.05 \\
0.0002 \\
0.004 \\
0.1 \\
0.005 \\
0.1 \\
0.01 \\
0.19 \\
0.556 \\
4 \\
1\end{array}$ & WS) $\begin{array}{c}<0.0017 \\
0.3907 \\
<0.0037 \\
<0.0456 \\
<1 \\
0.0267 \\
<0.0001 \\
3.83 \\
<0.0023 \\
<0.07 \\
<0.0001 \\
<0.0001 \\
<0.0002 \\
<0.002 \\
0.005 \\
0.005 \\
0.104 \\
<3.08 \\
382 \\
12.7\end{array}$ \\
\hline $\begin{array}{l}\text { Parameters establishing groundwater qualit } \\
\text { Chloride } \\
\text { Iron } \\
\text { Manganese } \\
\text { Phenols } \\
\text { Sodium } \\
\text { Sulfate }\end{array}$ & $\begin{array}{l}\text { NDb } \\
\text { ND } \\
\text { ND } \\
\text { ND } \\
\text { ND } \\
\text { ND }\end{array}$ & $\begin{array}{l}14.7 \\
12.1 \\
2.37 \\
<0.001 \\
22.8 \\
17\end{array}$ \\
\hline $\begin{array}{l}\text { Parameters used as indicators of groundwat } \\
\text { pH } \\
\text { Specific conductance, } \mu S / \mathrm{cm} \\
\text { Total organic carbon } \\
\text { Total organic ha? ogen }\end{array}$ & $\begin{array}{l}\text { er contam } \\
\text { ND } \\
\text { ND } \\
\text { ND } \\
\text { ND }\end{array}$ & ion $\begin{array}{l}6.49 \\
376 \\
6.37 \\
0.03\end{array}$ \\
\hline $\begin{array}{l}\text { Nonregulated parameters } \\
\text { PCBs } \\
\text { Tritium, } B q / \mathrm{L} \\
\text { Cesium- } 137, \mathrm{~Bq} / \mathrm{L} \\
\text { Strontium-90, Bq/L }\end{array}$ & $\begin{array}{l}\text { ND } \\
\text { ND } \\
\text { ND } \\
\text { ND }\end{array}$ & $\begin{array}{c}0.0001 \\
94333 \\
1.71 \\
223\end{array}$ \\
\hline
\end{tabular}

amean concentrations [in $m g / L$, unless otherwise noted (gross beta is in $\mathrm{Bq} / \mathrm{L}$ )] taken from three monitoring wells the first two quarters of 1985 (total of six analyses). Data taken from R. G. Stansfield and C. W. Francis, 1986, Characterization of the 01d Hydrofracture Facility (OHF) Impoundment, ORNL/TM-9990, Oak Ridge National Laboratory, Oak Ridge, Tennessee.

$$
b_{N D}=\text { not determined. }
$$




\subsection{GEOLOGY ANO SOILS}

\subsubsection{Regional Geology}

Dak Ridge National Laboratory lies in the Ridge and Valley Physiographic Province. In Tennessee, the province consists of northeast-southwest striking rock strata of limestone, sandstone, and shaie extending from the Virginia border on the north to the Georgia Alabama border on the south. The strata is tilted to angles of 30 degrees and greater throughout its length, resulting in the erosion-resistant beds forming parallel ridges, and those less resistant beds becoming intervening valley floors.

\subsubsection{Site Geology}

\subsubsection{Bedrock}

The DHF site lies in Melton Valley approximately $1200 \mathrm{~m}(4000 \mathrm{ft})$ southeast of the copper creek fault. As shown on the geologic map of Fig. 12, the site is underlain by unit "Ccb" of the conasauga Group. The lithology of this unit is described by Mc Master and waller (1965) as follows:

Variable lithology, ranging from shale and siltstone to limestone. Limestone is characteristically pebble conglomerate or edgewise conglonerate having irregular bedding surfaces coated with thin film of dark grey clay and marked by abundant ropy 'worm trails'. Limestone occurs in zones of shale and silestone. Siltstone in this unit is comonly calcareous and white or light grey when fresh. Shale is thinly bedded, colored brown, olive, and tan, and locally maroon. In places the unit is deformed by very small, sharp folds and faults of small displacement. 

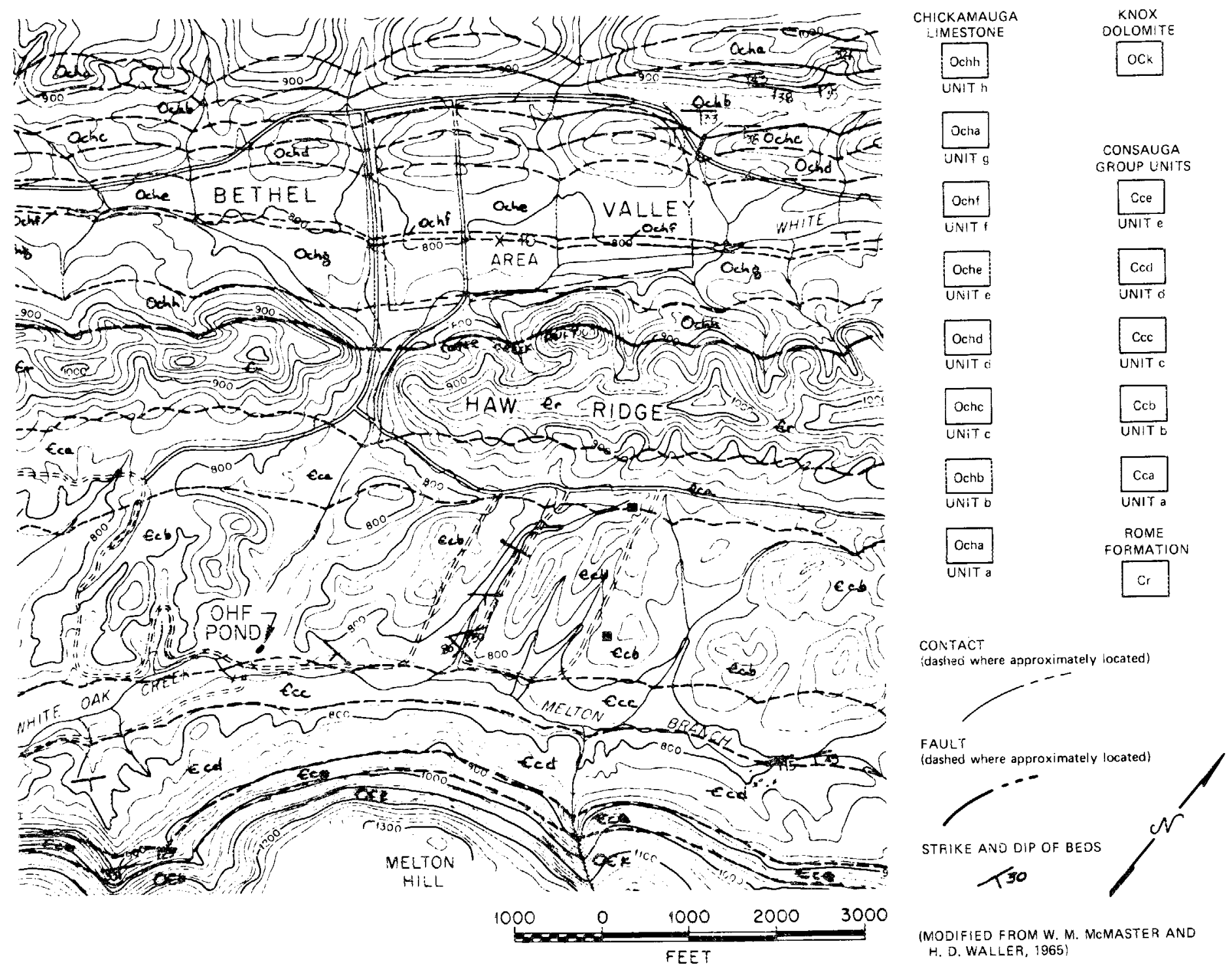

Fig. 12. Geologic map of the ORNL area including the old nydrofracture facility. 
They describe the residual material as follows:

Unit weathers to a bedded shale appearance, leaving little or no indication of original calcareous nature. Limestone weathers to porous brown siltstone or to a light orange-yellow illitic clay. Residuum is generally light tan to yellow-brown but local variations include maroon and green bands. Black manganese oxide stains common on joint surfaces.

OHF was constructed in the upper beds of the Ccb unit as mapped by McMaster and Waller (1965). From later work in the area by others (Haase et a1. 1985; Davis et al. 1984; and Rothschild et al. 1984) the upper beds of this mapped unit can be correlated with the upper portion of the Maryville Limestone Formation of the Conasauga Group. Davis and Stansfield (1984) reported on the excavation and construction for a French drain in the Maryville Formation at Solid Waste Storage Area (SWSA) 6, approximately $1200 \mathrm{~m}(4000 \mathrm{ft})$ southwest of the OHF site and along geologic strike. They found that the attitude of the beds vary locally from horizontal to dipping to the southeast as steep as 60 degrees due to folding of the strata. They also found two, near-vertical joint sets in the weathered rock. Slides into the french drain, along bedding planes, indicate that either a third joint system or bedding plane faults existed along those surfaces. Both vertical joint systems were very closely spaced (approximately $1 \mathrm{~cm}$ ) in the weathered rock. At the location that was subsequently the site of the OHF facility. Cowser et a1. (1961) mapped the bedrock strata as striking approximately north 43 degrees east, and dipping 15 degrees to the southeast. 
A geologic section through the impoundment is shown in Fig. 13 . The elevation of the sediment and bottom of the impoundment, as shown in the figure, is from Stansfield and Francis (1986).

\subsubsection{Soils}

The soil depth around the impoundment ranges from approximately 1.5 to $2.7 \mathrm{~m}(5$ to $9 \mathrm{ft})$. The soil consists of material which classifies as clay under the Unified Soil Classification System (Stansfield and Francis 1986). This clay soil overburden is the residuum of the underlying bedrock. Soils derived from the Conasauga Group contain illite and vermiculite as the predominant clay minerals (McMaster and Waller 1965).

\subsection{HYDROLOGY}

From 1948 through 1983, the mean annual precipitation at Oak Ridge was $138.71 \mathrm{~cm}(54.61 \mathrm{in})$. In this region, the heaviest precipitation normally occurs during winter and early spring, with the monthly maximum normally occurring during the period January to March. However, during some years the monthly maximum has occurred in July because of thunderstorms. September and october are usually the driest months. According to the Climatic Atlas of the United States (U.S Department of Commerce 1979), mean annual lake evaporation in the Oak Ridge area is $89 \mathrm{~cm}(33 \mathrm{in})$. From the above data it can be estimated that the net annual precipitation input to the DHF impoundment is $56 \mathrm{~cm}$ (22 in). Multiplying this amount by the surface area of the impoundment yields an average yearly retained precipitation contribution of approximately $140,000 \mathrm{~L}(37,000 \mathrm{gal})$. In late summer of 1985 , the water surface of 


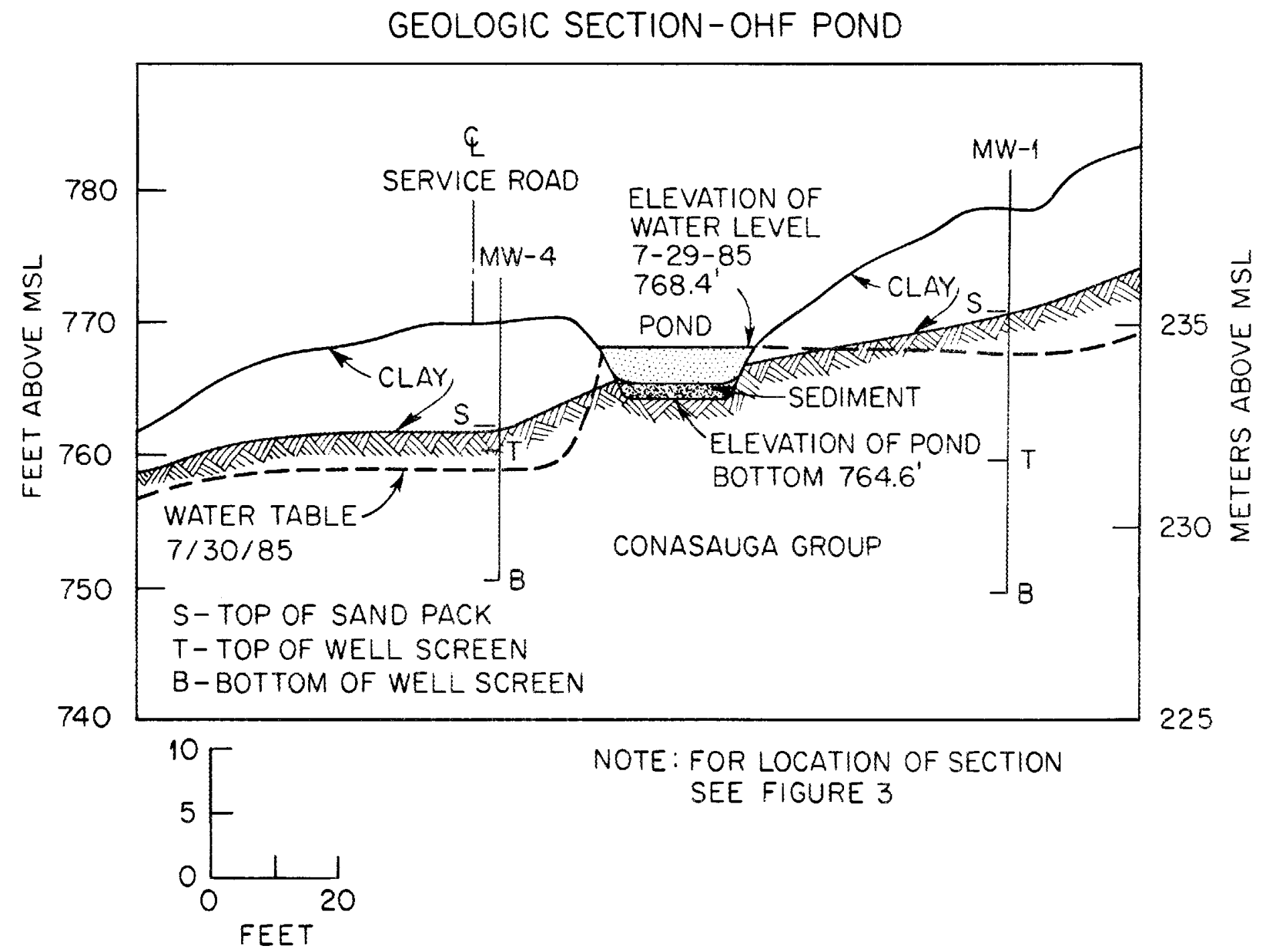

Fig. 13. Geologic section through the old hydrofracture facility impoundment. 
ORNL/TM-9991

the impoundment was measured at elevation $234.2 \mathrm{~m}(768.4 \mathrm{ft})$ above ms 1 . At this elevation, the capacity of the impoundment is approximately $300,000 \mathrm{~L}$ (77,000 gal). Therefore, the average net annual precipitation input amounts to slightly less than one-half the capacity of the impoundment at this level. The only design outflow system appears to be by the vertical standpipe, and this overflow system does not come into operation until slightly below the elevation at which overflow of the west wall of the impoundment occurs. As there have been no withdrawals of water from the impoundment since operation of the facility ceased, it appears that on a annual basis a net volume of approximately $140,000 \mathrm{~L}(37,000 \mathrm{gal})$ is leaking from the impoundment and entering the groundwater.

\subsubsection{Groundwater Movement}

Two water-table maps are shown in Figs. 14 and 15. The map in Fig. 14, from a report by Cowser et al (1961), depicts the water table for SWSA 5 prior to the construction of the OHF impoundment on the western border of the area. Figure 15 is based on water level observations from the four monitoring wells constructed in 1985 (Stansfield and Francis 1986) and is limited to the immediate site of the OHF impoundment. Water level observations upon which Fig. 15 is based are provided in Table 11. Both Figs. 14 and 15 show the hydraulic gradient at the impoundment to be generally towards white Oak creek, which is also the general direction of geologic strike of the bedrock strata. At the impoundment, as shown in Fig. 15, the gradient also has a component in the direction of Meiton Branch, which is in the general direction of the geologic dip of the bedrock strata. 


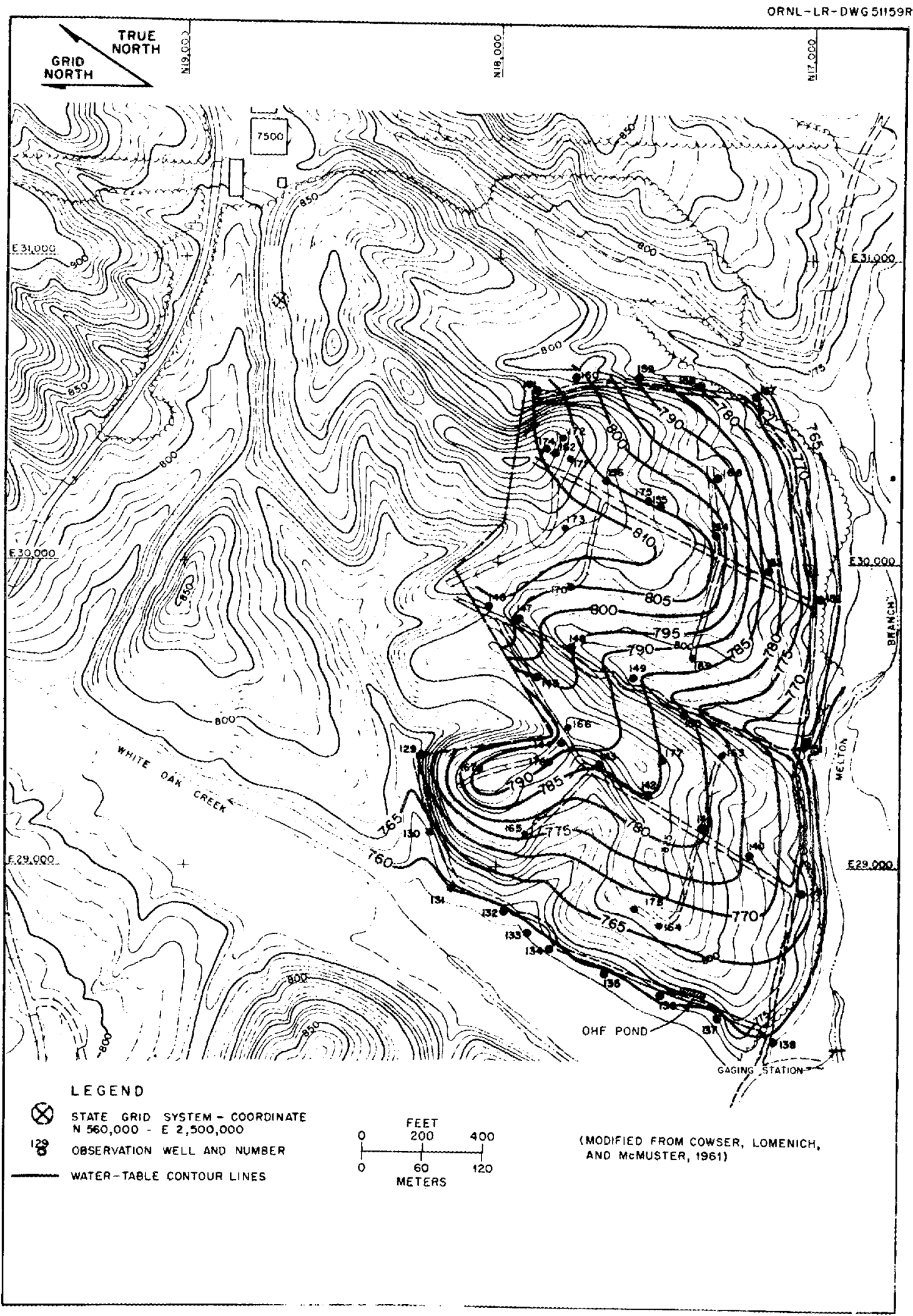

Fig. 14. General water-table map of the area upgradient from the old hydrofracture facility. 

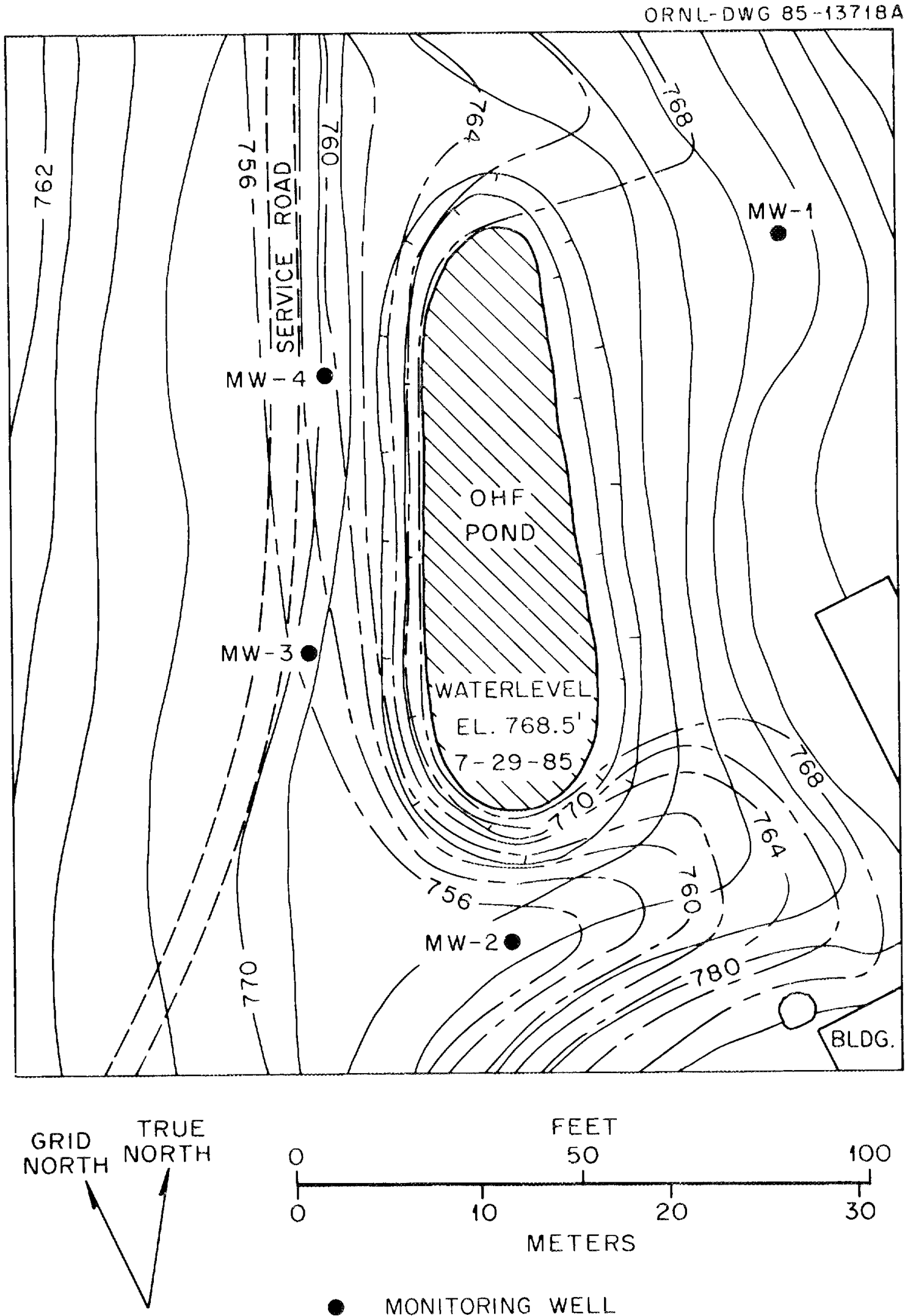

- monitoring well

WATER TABLE CONTOUR

Fig. 15. Water-table map of the old hydrofracture facility impoundment. 
As shown in the geologic section through the impoundment (Fig. 13), and the impoundment's water-table map (Fig. 15), the water table is below the bottom of the impoundment on the downgradient (west and south) sides. The water level on the upgradient side is several feet above the bottom of the impoundment. In the summer of 1985 , the water surface of the impoundment was approximately at the same elevation as the upgradient monitoring well. Studies on the ORNL reservation (Webster 1976; Davis et a1, 1984) show that in the bedrock, the direction of groundwater movement is greatly affected by the directional permeability of the strata. Therefore, the overall groundwater movement through the bedrock is often in a direction at some acute angle to the groundwater contours. The conasauga strata is anisotropic in respect to hydraulic conductivity with conductivities parallel to the direction of geologic strike being reported as 3 to 20 times greater than in a direction normal to the strike. Such movement would not normally be expected to be in a straight line of flow, but rather would follow irregular pathways along joints and bedding planes because the bedrock strata has very low primary permeability.

\subsubsection{Uppermost Aquifer}

The soil at the impoundment consists of material that has been visually classified as clay (according to the Unified soils Classification system), which categorically has a low hydraulic conductivity. However, as shown in Fig. 13, the tater table is below the soil, except at the edge of the impoundinent. Therefore, the uppermost aquifer at the site is the "CCb" unit (probably the 
Maryville Limestone formation). Davis et a1. (1984) conducted hydraulic conductivity tests in 36 monitoring wells in the Maryville Limestone. Conductivities ranged from $1 \times 10^{-5}$ to $238 \times 10^{-5} \mathrm{~cm} / \mathrm{s}$ with a geometrical mean of $6.31 \times 10^{-5} \mathrm{~cm} / \mathrm{s}$, and the effective porosity was estimated to be 0.03 . These aquifer characteristics are believed to be representative of the OHF site.

\subsection{ECOLOGY}

The OHF impoundment has not been a site for intensive radioecology studies such as those conducted at the 3513 impoundment (Garten 1982 , Kaye and Dunaway 1962, and Trabalka et al. in press). However, the flora and fauna occupying the OHF impoundment are generally considered similar to those in the 3513 impoundment. The major difference is that the OHF impoundment is considerably smaller than the 3513 impoundment. The oHf impoundment is located on a northwestern slope, surrounded by some trees, whereas the 3513 impoundment is located at the bottom of a small floodplain, making for some differences in the quantity and intensity of sunlight striking the impoundments. S. F. Huang (EOSO, personal communication, September 1984) remarked that the impoundment contained large quantities of filamentous algae and measured the level of cesium-137 in the algae to be approximately $60 \mathrm{kBq} / \mathrm{g}(2 \mathrm{uCi} / \mathrm{g})$ on the dry weight basis. 


\section{ADOITIONAL INFORMATION NEEDED}

In future phases of the ORNL remedial action program, the intent is to evaluate the transport and dose pathways for hazardous substances from each of the considered to pose an undue rish to health, safety, and environment facilities, using standard as well as state-of the -art mathematical models to provide adequate assessment of the site conditions. This pertains especially to the assessment of potential impacts on groundwater quality, which are difficult to determine using conventional analytical procedures. One purpose of this report is to identify the necessary data requirements to be used as input to drive such models. This pathway analysis assessment will then be utilized to determine the proper course of action required for future remedial actions.

\subsection{CONTAMINANT INVENTORY}

4.1.1. Buildings

4.1.1.1. Building 7852

Additional radiological survey in this building offers little benefit. Radiation levels in the control room, mixing cells, pump cel1, well cell, pump room, and engine pad are high; therefore, additional readings offer little new information. The radiation "shine" due to scatter would easily mask any moderate variations in levels of contaminants in the areas. Similarly, the transferable levels of radioactive contamination are high, and additional efforts at 
sampling would likely only redistribute the contamination within the area. Core samples of walls and floors could be obtained to determine the depth of penetration of radio-contaninants; however, the high levels of contamination on the surfaces could cause cross-contamination problems, making the interpretation difficult and casting doubts on any results that indicated penetration.

Some equipment remains in the cells and is presumably contaminated. For example, the Moyno pump in the pump room was observed to be contaminated. In the other cells, specific equipment was not identified as being contaminated. Thus, an additional survey in each of the cells should be conducted in an attempt to determine levels of contamination associated with particular pieces of equipment. Such a survey should be conducted only after initial decontamination work is completed. The high levels of transferable contamination observed in the initial survey indicate that a relatively simple cleaning of the surfaces of walls, floors, and equipment would greatly reduce radiation levels. At that time additional survey work could be done to deternine areas of residual contamination as well as to measure radiation levels on the equipment in the rooms. Verification of the isotopes involved needs to be undertaken, and it is especially important to determine if levels exceed TRU limits.

Outside building 7852, a radiological survey needs to be conducted to determine if the bulk storage bins are contaminated. The objective of this survey would be to determine if these bins, and their associated equipment, can be "green tagged" and thus released to salvage operations. The 97,000-L (25,000-gal) water tank and pump 
directly to the east of building 7852 should also be included in this survey to determine if these items can be salvaged.

\subsubsection{Building 7853}

This building as well as the equipment stored in the building needs to be surveyed to verify perceived noncontamination.

\subsubsection{Pump House}

In the initial survey made by $S$. F. Huang (EOSO, personal communication, September 1984), only a cursory survey was made of the valve pit. Therefore, an additional survey is required of this area. This would involve removing the sheet metal covering the valve pit and making radiation readings and taking smear samples from the interior of the pit. No surveys were made of the piping system of the OHF. Thus, the valve pit area would be a good place to disconnect some of the piping and determine the levels of contamination on the interior surfaces of the pipe. A complete survey of the OHF piping system would require a great deal of excavation and expense; on the other hand, analyzing the contamination present on the outside as well on the interior of the pipes in the valve pit area would be much more practical than the extensive excavation of the pipelines. To determine whether extensive leakage from pipes has occurred, however, soil cores need to be taken to depths below the pipes and analyzed for radioactivity. Perhaps pressure tests on specific pipelines could be used to determine suspected leakage. 


\subsubsection{Waste Pits}

Additional analyses, both radiological and for RCRA hazardous constituents (using the RCRA-EP leach test), need to be conducted on the solidified material at the bottom of the compartments already surveyed by S. F. Huang (EOSO, personal communication, September 1984) and on the contents of the sealed third compartinent. This will involve taking drilled core samples from the concrete-like material. Sections of these cores will be analyzed and estimates made as to total inventory of radionuclides and hazardous constituents. Radiological analyses will also include analyses for transuranics to determine if these materials contain TRU levels in excess of the TRU waste handing limits.

\subsubsection{Waste Tanks}

These tanks were not surveyed directly in the S. F. Huang (EOSD, personal cominunication, September 1984) survey. For example, the estimated inventories of up to $37 \mathrm{TBq}$ ( $1 \mathrm{kCi})$ per tank were made based on past operating experience of how much and the character of waste left in the tank. Thus, detailed characterization of the sludge as to quantity and activity levels is needed prior to removal of the tanks. To do this, the soil covering the tanks would need to be removed so that access to the tank could be made to measure the quantity of sludge remaining and to take samples for radiological and RCRA analyses. It is not clear from the available documentation if portals on top of the tank exist so that such sampling can be conducted. Additional soil cores adjacent these tank also need to be taken, as there was an indication of elevated levels of gamma activity in the core sample 
(number 16, directly southwest of tank $r-4$ ) taken by $S$. F. Huang (EOSO, personal communication, September 1984) from around the tanks. Further characterization of soil around the tanks is addressed in sect. 5.2.2.

\subsubsection{Waste Impoundment}

The studies of S. F. Huang (EOSO, personal communication, September 1984) and Stansfield and Francis (1986) provided an adequate data base to determine the inventory of both radionuclides and nonradioactive contaminants for the pond water and sediment. However, additional samples of subsurface soils need to be taken from the berm as well as from underneath the impoundment to determine the extent of contamination, both in terms of concentration and soil depth. Also, if the modeling predictions associated with remedial action are truly dependent on being able to model the extent of the contamination under existing conditions, then leaching studies on the sediment need to be conducted. The leaching should be conducted with pond water from the impoundment, and any subsequent attenuation of the transported contaminants by the soil underlying the impoundment and subsurface surrounding soils should also be determined. Sediment samples need to be taken to assure that any engineering approaches to the remedial actions, such as stabilizing the sediment, can be completed.

\subsection{GEOLOGY AND SOILS}

\subsubsection{Site Geology}

The first step in further characterization will be coring at depths ranging from 20 to $40 \mathrm{~m}(65$ to $130 \mathrm{ft}$ ) to determine if deep soils and bedrock are contaminated. For example, core sections will be 
analyzed for radioactive as well as nonradioactive contamination (using the RCRA EP leach test). Cores of this type serve as excellent integrators of past contamination. One core will be taken between the impoundment and the waste tanks, and another in a southwesterly direction downgradient to the facility.

If the bedrock is found to be contaminated, further efforts will be needed to determine the bedding plane characteristics and their frequencies so that the extent of contamination can be modeled. To observe bedding plane characteristics and frequency in the conasauga, three exploratory borings of approximately a $33-\mathrm{m}(100-\mathrm{ft})$ depth would be needed. As a major proportion of the joint sets are believed to be in the near vertical attitude, the jointing frequency of the major proportion of joint sets will not be obtained from a vertical core nole. Therefore, two of the exploratory borings will be inclined from the vertical at 30 degrees or more and at right angles to each other. A likely location of the core holes would be at the southwest corner of the impoundment. Selected core samples should be prepared for geochemical analysis including stable isotope and fluid inclusion analysis. These actions, however, are dependent on verification of contamination in the bedrock.

\subsubsection{Soils}

Soil samples need to be taken so that sorption/desorption of known contaminants in the pond water and sediment on the subsoils below and around the impoundment can be determined. To validate modeling of existing conditions, detailed spatial concentrations in soils 
near-field the impoundment will be needed. For example, the possibility of seepage from the impoundment having occurred in the subsurface, along the 8 -in $(20-\mathrm{cm})$ diam drain line installed at the northwest corner of the impoundment to act as an emergency overflow, needs to be investigated. This will require coring along the pipeline and analyses of soil to check for contamination. The same pattern of soil coring needs to be conducted along pipe lines from the waste storage tanks to building 7852 , and from building 7852 to the impoundment and waste pits (see fig. 10).

In the surveying of the waste tanks, soil excavated to reach the tanks needs to be sampled and analyzed for radioactivity and RCRA constituents (via use of the RCRA EP leach test). In addition, soil cores from between the tanks should be taken and analyzed to determine the extent of contamination.

\subsection{HYOROLOGY}

The results of analyses and groundwater-level measurements from the monitoring wells constructed in the fall of 1985 need to be evaluated in concert with the several piezometer wells that are scheduled for completion in the area of the impoundment in 1986. A potentiometric map for the area will be prepared by using data from all wells.

\subsubsection{Surface Water}

To model the impoundment, at least monthly water level observations of the impoundment and monitoring wells should be recorded for one year. A water budget will need to be made for the impoundment to determine the leakage from the impoundment. 


\subsubsection{Groundwater}

Groundwater samples, from the OHF impoundment monitoring wells, have been taken and analyzed four times during the period starting in February 1985 and ending in September 1985. These results need to be evaluated to determine whether contaminants other than radionuclides from the impoundment have significantly affected the groundwater. Pollution from chemical hazardous waste is assumed if an analysis by a statistical procedure such as Cochran's Approximation to the Behrens-Fisher Student's $t$ test indicates a significant increase (decrease in the case of $\mathrm{pH}$ ) in the water quality parameters listed in Table 12 between the upgradient and downgradient wel1s.

If contamination has been verified deep into the weathered bedrock by the $20-$ to $40-m(60-$ to $130-f t)$ corings (see sect. 4.2.1.), additional data on the aquifer properties (hydraulic conductivity, effective porosity, and degree of anisotropism) will be needed for constructing models of the groundwater flow system and contaminant transport. Hydraulic conductivity tests, using slug-test procedures, will be conducted and analyzed for the existing monitoring wells at the impoundment. A pump test with several observation wells will be necessary to study the anisotropic nature of the hydraulic conductivity and transmissivity. The well to be pumped in the test will need to be at least $10 \mathrm{~cm}$ in diameter so that a submersible pump can be installed. Because of this relatively large diameter, rather than drill the well using coring techniques, normal well drilling methods will be used. The strata in the well will be examined and logged by the use of a down-the-hole camera. 
Additional shallow-groundwater monitoring wells will be needed on the west side of the OHF impoundment to define the limits of contamination. Wells will have to be sufficient in number and depth to determine the lateral extent of the contamination. At present, the groundwater adjacent to the concrete block pits and carbon steel waste tanks is not monitored. It will therefore be necessary to construct additional shallow-groundwater monitoring wells for this purpose [depths of wells up to approximately $10 \mathrm{~m}(32 \mathrm{ft})$ ]. This will require three wells downgradient in a semicircle surrounding the southwest corner of the facility, but at further distances than the wells presently surrounding the impoundment. Two wells will also be constructed upgradient to the impoundment and waste pits to differentiate any contamination being released from SWSA 5 burial ground immediately to the northeast. The drill cuttings will need to be monitored for radioactivity during the well drilling.

The monitoring of groundwater at depths greater than $10 \mathrm{~m}(32 \mathrm{ft})$ is presently being conducted by the U.S. Geological Survey (Webster 1982). One of the monitoring stations is approximately $15 \mathrm{~m}(48 \mathrm{ft}$ ) to the northwest of the facility. If analysis of deep cores indicate contamination (see sect. 4.2.1.), then one or more deep-groundwater monitoring wells will be constructed.

\subsection{ECOLOGY}

Before implementation of any remedial action, a biological survey documenting the flora and fauna present in the OHF impoundment should be conducted. The intent here is to determine if the aquatic 
communities in the pond are similar to those found in the 3513 impoundment where extensive radioecological studies have been conducted (see Sect. 4.4). 


\section{MONITORING AND TESTING PLAN}

\subsection{RECOMMENDED TASKS}

The following is a list of specific tasks that need to be accomplished to determine the remedial actions needed:

1. Conduct a Radiological Survey of Equipment in Building 7852 After washing, with a strong cleaning solution, surfaces of walls and floors of rooms in building 7852 that showed high surface contamination, a radiological survey of equipment in building 7852 will be conducted.

2. Collect and Analyze Materials Stored in Waste pits Samples of waste from the three waste pits will be taken and analyzed for radionuclide and RCRA hazardous waste concentrations to estimate total inventory of these constituents.

3. Measure Concentrations of Radionuclides and RCRA Hazardous Waste in Waste Tanks

Representative samples of waste will be taken from all five steel waste tanks and the quantity of waste in each measured. The purpose is to determine concentrations as well as total inventory of radionuclides and RCRA hazardous waste constituents in each of the tanks. To do this, the soil overlying these tanks must be excavated.

4. Conduct a Radiological Survey of Valve Pit and Associated OHF Piping The valve pit, adjacent to the pump house, has never been thoroughly surveyed for radioactive contaminants. The covering of the valve pit needs to be removed and a radiological survey conducted. In addition, piping in this pit needs to be 
disassembled and activity on the interior of the piping determined to evaluate the potential activity related to the underground piping throughout the OHF. Soil cores will be taken and analyzed down to and along known areas of underground piping to determine if leakage has occurred. This includes the emergency overflow pipeline from the impoundment, the two influent pipelines to the impoundment, as well as other pipelinesfrom the waste tanks and waste pit. For soil samples that show contamination with radionuclides (indicating a pipeline leak), the EP leach test will be used to assess the soil's RCRA hazard characteristic.

5. Perform Statistical Analysis of Groundwater Monitoring Data Using an adequate statistical analysis procedure, one year of groundwater monitoring data will be evaluated (samples collected over four quarters and analyzed for the parameters established by the U.S. Environmental Protection Agency as indicators of significant groundwater contamination by chemical hazardous waste).

6. Conduct Sediment Leaching and Soil Attenuation Studies

Studies to determine the leaching rates of containinated sediment by pond water in conjunction with sorption/desorption attenuation studies of the leached contaminants on berm soils and soil samples taken from below the pond sediment are needed. 
7. Determine Impoundment Leakage

A water budget for the impoundment will be determined by monitoring pond water and groundwater elevations on a monthly basis. Rainfall and free water evaporation rates will be determined at the site. Dye and or short-lived radionuclide tracer tests will also be conducted. For example, the loss from the impoundment will be estimated by comparing the concentration of the tracer in the impoundment over time. Also, the direction of flow and leakage to white 0ak creek can likely be determined.

8. Determine Lateral Limits of Shallow-Groundwater Contamination

Additional groundwater monitoring wells will be required to determine the lateral limits of shallow-groundwater contamination. This includes three additional wells that encompass the southern and western boundaries of the OHF impoundment and two wells upgradient between the facility and SWSA 5. The 2-in-diam wells will be constructed of stainless steel.

9. Determine Vertical Limits of Contamination Vertical contamination deep into the partially weathered bedrock (30 m or more) will be determined by extracting two corings from the site. Tentatively, the location of one core will be at the southwest corner of the impoundment but close to the waste tanks. The other core should be taken downgradient in a southwesterly direction. Sections of these cores will be surveyed for radionuclides, and samples will be taken and extracted using the RCRA EP leaching test to determine the presence of RCRA hazardous chemicals. 
10. Collect and Analyze Data from New Monitoring Wells

Sampling and analyses of the new wells for gross alpha, gross

beta, Sr-90, and H-3 is planned. Also, if the statistical

analyses indicates significant pollution by chemical waste,

then all groundwater samples for all RCRA constituents will

be analyzed.

11. Conduct Single-Well Hydraulic Conductivity Tests

Single-well hydraulic conductivity tests (slug tests) will be conducted in all monitoring wells and the respective hydraulic conductivities of the surrounding geologic media will be calculated.

12. Conduct an Ecological Survex of oHf Impoundment

A survey will be conducted to determine the major biological fauna and flora living in the OHF impoundment.

13. Prepare Pathway Analys is

Data from the presently available characterization information and from that obtained from the above actions will be used to prepare a pathway analysis of contaminants from the facility using documented models.

14. Coordinate Project and Prepare Report

This action includes the overall coordination role to complete the above tasks, as well as to take the information obtained from the above tasks and to document them in a report 


\section{REFERENCES}

Cowser, K. E., T. F. Lominick, and W. M. McMaster. 1961. Status Report on Evaluation of Solid Waste Disposal at ORNL. ORNL-3035. Oak Ridge National Laboratory, Oak Ridge, Tennessee.

Davis, E. C., W. J. Boegly, Jr., E, R, Rothschild, B. P. Spalding, N. D. Vaughan, C. S. Haase, D. D. Huff, S. Y. Lee, E. C. Walls, J. D. Bold, and E. D. Smith. 1984. Site characterization Techniques Used at a Low-level Waste Shallow Land Burial Field Demonstration Facility. ORNL/TM-9146. Dak Ridge National Laboratory, Oak Ridge, Tennessee.

Davis, E. C. and R. G. Stansfield. 1984. Design and Construction of a French Drain for Groundwater Diversion in Solid Waste Storage Area Six at the OAK Ridge National Laboratory. ORNL/TM-9014. Oak Ridge National Laboratory, Oak Ridge, Tennessee.

Garten, C. T. 1982. Comparative food chain behaviour and distribution of actinide elements in and around a contaminated fresh-water pond. pp. 299-312. In Environmental Migration of Long-Lived Radionuclides. International Atomic Energy Agency, Vienna.

Gupton, E. D. 1961. Health Physics Instrument Manual. ORNL/TM-332.

Oak Ridge National Laboratory, Oak Ridge, Tennessee.

Haase, C. S., C. L. Zucker, and S. H. Stow. 1985. Geology of the Host Formation for the New Hydrofracturing Facility at Oak Ridge National Laboratory. Proceedings of Waste Management 85.

R. G. Post and M. E. Wacks, Editors, Tucson, Arizona, March 23-28, 1985. 
Kaye, S. V. and P. B. Dunaway. 1962. Bioaccumulation of radioactive isotopes by herbivorous small mammals. Health Phys. 7:205-217.

de Laguna, W., H. 0. Weeren, F. T. Binford, E. J. Witkowski, and E. G. Struxness. 1971. Safety Analysis of Waste Disposal by Hydraulic Fracturing at Dak Ridge. ORNL-4665. Oak Ridge National Laboratory, Oak Ridge, Tennessee.

McMaster, W. M. and H. D. Waller. 1965. Geology and Soils of White Dak Creek Basin, Tennessee. ORNL/TM-1108. Oak Ridge National Laboratory, Oak Ridge, Tennessee.

Myrick, T. E. 1984. The ORNL Surplus Facilities Management Program Long-Range Program. ORNL/TM-8957. Oak Ridge National Laboratory, Oak Ridge, Tennessee.

Rothschild, E. R., D. D. Huff, C. S. Haase, R. B. Clapp, 8. P. Spalding, C. D. Farmer, and N. D. Farrow. 1984. Geohydrologic Characterization of Proposed Solid Waste Storage Area (SWSA) 7. ORNL/TM-9314. Oak Ridge National Laboratory, Oak Ridge, Tennessee. Spaiding, B. P. and I. L. Munro, 1984. Determination of the Areal Distribution of ${ }^{90} \mathrm{Sr}$ in Groundwater via single-Use Boreholes. ORNL/TM-8991. Oak Ridge National Laboratory, Oak Ridge, Tennessee. Stansfield, R. G. and C. W. Francis. 1986. Characterization of the 0ld Hydrofracture Facility (OHF) Impoundment. ORNL/TM 9990. Oak Ridge National Laboratory, Oak Ridge, Tennessee. Trabalka, J. R., M. A. Bogle, E. A. Bondietti, and T. G. Scott. In press. Actinide behavior in a freshwater pond. In symposium on Environmental Research for Actinide Elements. J.E. Pinder, III, J. J. Alberts, K. W. MC Cloud, and R. G. Schreckhise (Eds.), November 7-11, 1984, Hilton Head, South Carolina. 
U.S. Department of Commerce. 1979. Climatic Atlas of the United States.

U.S. Environmental Protection Agency (USEPA). 1980. Identification and Listing of Hazardous Waste. In Environmental Protection Agency Hazardous Waste Management System, 40 CFR 261.24.

U.S. Environmental Protection Agency (USEPA). 1982. Test Methods for Evaluating Solid Waste, Physical/Chemical Methods, SW-846, 2nd Edition. Solid waste and Emergency Response, Washington, DC.

U.S. Environmental Protection Agency (USEPA). 1983. Permit Writers' Guidance Manual for Subpart F, Ground-Water Protection, Oraft. GeoTrans, Inc., Washington, DC.

Webster, 0. A. 1976. A Review of Hydrologic and Geologic Conditions Related to the Radioactive Solid Waste Burial Grounds at Dak Ridge National Laboratory, Tennessee. U.S. Geological Survey, Open-File Report No. $76-127$.

Webster, D. A. 1982. Water-Level Data for Wells in Burial Ground 5 , Dak Ridge National Laboratory, Tennessee, 1976-1980:

U.S. Geological Survey, Open-file Report No. 82-372.

Weeren, H. 0. 1976. Shale Fracturing Injections at Oak Ridge National Laboratory--1975 Series. ORNL/TM-5545. Oak Ridge National Laboratory, Oak Ridge, Tennessee.

Weeren, H. 0. 1980. Shale Fracturing Injections at Oak Ridge. National Laboratory--1977-1979 Series. ORNL/TM-7421. Oak Ridge National Laboratory, Oak Ridge, Tennessee. 

INTERNAL DISTRIBUTION

1. T. L. Ashwood

2. S. I. Auerbach

3. J. S. Baldwin

4-9. T. W. Burwinkle

10. R. B. Clapp

11. N. H. Cutshall

12. E. C. Davis

13. L. R. Dole

14. R. B. Dreier

15-19. C. W. Francis

20. C. S. Haase

21. S. G. Hildebrand

22. F. J. Homan

23. D. D. Huff

24. C. G. Jones

25. R. Jordan

26. R. H. Ketelle

27 L. W. Long

28. L. E. McNeese

29. F. R. Mynatt
30-39. T. E. Myrick

40. T. W. Dakes

41. C.R. Olsen

42. D. E. Reichle

43. T. H. Row

44. E. D. Smith

45. B. P. Spalding

46-50. R. G. Stansfield

51. S. H. Stow

52-61. L. E. Stratton

62. 3. Switek

63. G. T. Yeh

64. H. E. Zittel

65. Central Research Library

66-80. ESD Library

81-82. Laboratory Records Dept.

83. Laboratory Records, ORNL-RC

84. ORNL Patent Section

85. ORNL Y-12 Technical Library

\section{EXTERNAL DISTRIBUTION}

86. M. Barainca, Program Manager, Low-Level Waste Management Program, U.S. Department of Energy, 550 Second Street, Idaho Falls, ID 83401

87. J. J. Blakeslee, Program Manager, Nuclear Waste Processing, Rocky Flats Plant, Rockwell International, P.0. Box 464, Golden, C0 80401

88. J. Thomas Callahan, Associate Director, Ecosystem Studies Program, Room 336, $1800 \mathrm{G}$ Street. NW, National Science Foundation, Washington, DC 20550

89. T. C. Chee, R\&D and Byproducts Division, DP-123 (GTN), U.S. Department of Energy, Washington, DC 20545

90. A. T. Clark, Jr., Advanced Fuel and Spent Fuel Licensing Branch, Divison of Fuel Cycling and Material Safety, 396-SS, U.S. Nuclear Regulatory Commission, 7915 Eastern Avenue, Silver Spring, MD 20910

91. Peter Colombo, Group Leader, Nuclear Waste Research, Brookhaven National Laboratory. B1dg. 701, Upton, NY 11973

92. E. F. Conti, Office of Nuclear Regulatory Research, Nuclear Regulatory Commission, MS-1130-SS, Washington, DC 20555

93. J. E. Dieckhoner, Acting Director, Operations and Traffic Division, DP-122 (GTN), U.S. Department of Energy, Washington, DC 20545

94. G. J. Foley, Office of Environmental Process and Effects Research, U.S. Environmental Protection Agency, 401 M Street, SW, RD-682, Washington, DC 20460 
95. Carl Gertz, Director, Radioactive Waste Technology Division, Idaho Operations office, U.S. Department of Energy, 550 Second Street, Idaho Falls, I0 8340?

96. C. R. Goldman, Professor of Limnology. Director of Tahoe Research Group, Division of Environmental Studies, University of California, Davis, CA 95616

97. W. H. Hannum, Director, West Valley Project office, U.S. Department of Energy, P.0. Box 191, West Valley, NY 14171

98. J. W. Huckabee, Manager, Ecological Studies Program, Electric Power Research Institute, 3412 Hillviem Avenue, P.0. Box 10412, Palo Alto, CA 94303

99. E. A. Jennrich, Program Manager, Low-Level Waste Management Program, EG\&G Idaho, Inc., P.0. Box 1625, Idaho Falls, ID 83475

100. J. J. Jicha, Director, R\&D and Byproducts Division, DP-123 (GTN), U.S. Department of Energy, Washington, DC 20545

101. E. A. Jordan, Low Leve1 Naste Program Manager, Division of Storage and Treatment Projects, NE-25 (GTN), U.S. Department of Energy, Washington, OC 20545

102. George Y. Jordy, Director, Office of Program Analysis, Office of Energy Research, ER-30, G-226, U.S. Department of Energy. Washington, DC 20545

103. L. T. Lakey, Waste Isolation, Pacific Northwest Laboratory, Richland, WA 99352

104. Leonard Lane, Los Alamos National Laboratory, P.0. $80 \times 1663$. Los Alamos, NM 87545

105. D. B. Leclaire, Director, Office of Defense waste and Transportation Management, DP-12 (GTN), U.S. Department of Energy, Washington, DC 20545

106. Helen McCammon, Director. Ecological Research Division, office of Health and Environmental Research, office of Energy Research, MS-E201, ER-75, Room E-233, Department of Energy, Washington, DC 20545

107. Michael McFadden, Waste Management, Albuquerque Operations office, U.S. Department of Energy, Albuquerque, NM 37115

108. Edward 0'Donnel1, Division of Radiation Programs and Earth Sciences, U.S. Nuclear Regulatory Commission, Mail Stop 1130 SS, Washington, DC 20555

109. J. W. Patterson, Program Director, Waste Management Program Office, Rockwell Hanford Operations, P.D. Box 800, Richland, WA 99352

110. E. M. Romney, University of California, Los Angeles, 900 veteran Avenue, Los Angeles, CA 90024

111. Ilkka Savolainen, Waste Management Section, International Atomic Energy Agency, Wagramerstrasse 5, P. 0. Box 100, A-7 400 Vienna, Austria

112. R. J. Starmer, HLW Technical Development Branch, Office of Nuclear Material Safety and Safeguards, Nuclear Regulatory Commission, Room 427-SS, Washington, DC 20555

113. R. J. Stern, Director, Office of Environmental Compliance, MS PE-25, FORRESTAL, U.S. Department of Energy. 1000 Independence Avenue, Sw, Washington, DC 20585

114. M. T. Stewart, University of South Florida, Tampa, FL 33620 
115. J. A. Stone, Savannah River Laboratory, E. I. DuPont de Nemours and Company, Bidg. 773-A, Room E-112, Aiken, SC 29808

116. S. B. Upchurch, University of South Florida, Tampa, FL 33620

117. Leonard $H$. Weinstein, Program Director of Environmental Biology, Cornell University, Boyce Thompson Institute for Plant Research, Ithaca, NY 14853

118. Raymond G. Wilhour, Chief, Air Pollution Effects Branch, Corvallis Environmental Research Laboratory, U.S.

Environmental Protection Agency, 200 SW 35th Street, Corvallis, OR 97330

119. Frank J. Wobber, Ecological Research Division, Office of Health and Environmental Research, Office of Energy Research, MS-E201, Department of Energy, Washington, DC 20545

120. M. Gordon Wolman, The Johns Hopkins University, Department of Geography and Environmental Engineering, Baltimore, MD 21218

121. H. H. Zehner, U.S. Geological Survey-Water Resources Division, $1013 \mathrm{~N}$. Broadway, Knoxville, TN 37917

122. Office of Assistant Manager for Energy Research and Development, Oak Ridge Operations, P. 0. Box E, U.S. Department of Energy, Oak Ridge, TN 37831

123-149. Technical Information Center, Oak Ridge, TN 37831 